OPEN ACCESS

Edited by:

Amy Lovett-Racke,

The Ohio State University,

United States

Reviewed by:

Jun-ichi Kira,

Kyushu University, Japan

Klaus Dornmair,

Ludwig Maximilian University of

Munich, Germany

*Correspondence:

Jorge Correale

jcorreale@fleni.org.ar;

jorge.correale@gmail.com

Specialty section:

This article was submitted to

Multiple Sclerosis and

Neuroimmunology,

a section of the journal

Frontiers in Immunology

Received: 09 August 2019 Accepted: 31 October 2019 Published: 20 November 2019

Citation:

Carnero Contentti E, Farez MF and Correale J (2019) Mucosal-Associated

Invariant T Cell Features and TCR Repertoire Characteristics During the

Course of Multiple Sclerosis.

Front. Immunol. 10:2690.

doi: 10.3389/fimmu.2019.02690

\section{Mucosal-Associated Invariant T Cell Features and TCR Repertoire Characteristics During the Course of Multiple Sclerosis}

\author{
Edgar Carnero Contentti ${ }^{1}$, Mauricio F. Farez ${ }^{2}$ and Jorge Correale ${ }^{2,3 *}$ \\ ${ }^{1}$ Neuroimmunology Unit, Department of Neuroscience, Hospital Alemán, Buenos Aires, Argentina, ${ }^{2}$ Centro para el Estudio \\ de Enfermedades Neuroinmunologicas (CIEN), Fundación para la Lucha contra las Enfermedades Neurológicas de la \\ Infancia (FLENI), Buenos Aires, Argentina, ${ }^{3}$ Department of Neurology, Fundación para la Lucha contra las Enfermedades \\ Neurológicas de la Infancia (FLENI), Buenos Aires, Argentina
}

Objective: To investigate the frequency, phenotype, function, and longitudinal repertoire of mucosal-associated invariant T (MAIT) cells in relapsing remitting multiple sclerosis (RRMS) and primary progressive multiple sclerosis (PPMS) patients.

Methods: Forty-five RRMS patients in remission, 20 RRMS patients experiencing exacerbations, 15 PPMS patients, and 30 healthy controls (HCs) were included in the study. MAIT cells were identified phenotypically as $\mathrm{CD}^{+} \mathrm{TCR} \gamma \delta^{-} \mathrm{V} \alpha 7.2+\mathrm{CD} 161^{\text {high }}$. In 15 patients, MAIT cell number and MRI lesions were evaluated every 6 months, for 36 months. MAIT cell TCRV $\beta$ repertoire was defined using single-cell cloning and mRNA sequencing.

Results: Circulating MAIT cells were significantly reduced in both RRMS and PPMS patients, particularly during exacerbations, compared to healthy subjects. This decrease was accompanied by pro-inflammatory cytokine production (TNF- $\alpha$, IFN- $\gamma, \mathrm{IL}-17$, and GM-CSF). Three months post-exacerbation, peripheral blood MAIT cell percentages increased significantly along with clinical recovery. Likewise, we observed inverse correlation between MRI lesions and peripheral blood MAIT cell numbers. In paired samples, MAIT cell percentage was significantly higher in CSF than in peripheral blood, suggesting MAIT cell migration through the blood-brain barrier. Finally, MAIT cells showed limited TCRV $\beta$ repertoires, in both CSF and peripheral blood, which remained stable over time.

Conclusions: MAIT cell levels correlated with MS course both clinically and radiologically, showing marked and sustained oligoclonality. These findings may contribute to a better understanding of pathophysiological phenomena underlying the course of MS, and discovery of MAIT cell inhibitors could pave the way for the development of new therapeutic strategies.

Keywords: MAIT cells, multiple sclerosis, neuroimmunology, MRI, CSF (cerebrospinal fluid) 


\section{INTRODUCTION}

Although the etiology of multiple sclerosis (MS) remains elusive, it is now known that environmental factors and susceptible genes are involved in disease pathogenesis, with immunological, genetic, and histopathology studies of MS patients showing that autoimmunity plays a key role (1-3). In addition to adaptive $\mathrm{T}$ and B cell immune responses (4), recent studies have identified new populations of non-conventional $\mathrm{T}$ cells called invariable natural killer $\mathrm{T}$ (iNKT) cells and mucosal-associated invariant $\mathrm{T}$ (MAIT) cells, which function at the interface between adaptive immunity and innate immunity, and also play a role in different autoimmune (3-6). In humans, MAIT cells display a restricted $\alpha \beta$ T cell receptor (TCR), in which the TCRV $\alpha$ chain comprises a canonical V $\alpha 7.2-\mathrm{J} \alpha 33$ (from now on the IMGT denomination will be used: TRAV1-2-TRAJ33) rearrangement, paired with a limited number of TCR $\beta$ chains: TRBV20-1, TRBV6-1, TRBV64, TRBV6-5, and TRBV-13 $(7,8)$, and less frequent usage of the non-canonical TRAV1-2-TRAJ12/20 TCR rearrangement $(8,9)$. They can be identified by staining for TRAV1-2 and either CD161, or IL-18R $\alpha$, in the TCR $\gamma \delta^{-} \mathrm{CD}^{-} \mathrm{CD}^{+}$compartment (10). MAIT cells are restricted by MR1, a non-polymorphic class Ib-related MHC molecule (11), and recognize a limited antigen repertoire, represented by molecules that are precursors or derived from riboflavin and the metabolism of folic acid in bacteria and yeast $(12,13)$. Until now, no autoantigen recognized by MAIT cells has been reported. However, recent studies indicate that in addition to MAIT cells that recognize riboflavin- and folate-related metabolites, human blood contains MR1-restricted T cells, which recognize cell-derived antigens, participating in different inflammatory mechanisms involving B cells and dendritic cells. (14). These data expand the function of MR1 beyond the presentation of microbial antigens, indicating that MR1-restricted cells can participate in the development of different inflammatory processes.

MAIT cells are abundant in tissues exposed to microbial antigens, including lamina propria of the gut and liver, important sites of host-environment interactions. They can be activated in an MR1-dependent or -independent manner. After activation, MAIT cells proliferate, secreting pro-inflammatory cytokines including IFN- $\gamma$, TNF- $\alpha$, GM-CSF, and IL-17, as well as cytotoxic effector molecules such as perforin and granzymes (15-18), resulting in lysis of infected cells (19). These properties suggest that MAIT cells may function as first responders to aberrant microbial signals, while also sustaining abnormal inflammatory reactions (19).

In adult humans, MAIT cells represent up to $10 \%$ of circulating $\mathrm{T}$ cells, $20-45 \%$ of $\mathrm{T}$ cells in the liver, and $3-5 \%$ of lymphoid cells in the intestinal mucosal $(20,21)$. Currently, although their preferential distribution at various mucosal sites indicates a role in host responses at the site of pathogen entry, little is known about the roles of these cells in immune-mediated diseases, such as MS (10). MAIT cells have been found in CNS lesions from MS patients $(22,23)$, which raises questions on their function within the CNS and their relevance in disease pathogenesis. Contradictory observations have been made in different MS cohorts, reporting either a protective or deleterious effect of MAIT cells as well as in experimental autoimmune encephalomyelitis (EAE) (23-28).

In the present study, we investigated frequency, phenotype, function, and longitudinal repertoire of MAIT cells in relapsing remitting and primary progressive MS patients. The multifaceted nature of MAIT cells makes them promising candidates both for therapeutic targeting and as potential biomarkers of disease.

\section{METHODS}

\section{Study Design and Patient Selection}

Eighty-six patients diagnosed with clinically definitive MS were recruited through the MS Clinic at the Raúl Carrea Institute for Neurological Research, FLENI. MS was diagnosed according to 2010 revised McDonald criteria. Patients were divided into three groups: (i) relapsing remitting MS in remission (RRMS; $n=$ 46), (ii) relapsing remitting MS experiencing acute exacerbations (RREMS; $n=25$ ), and (iii) primary progressive MS cases (PPMS; $n=15$ ). Exacerbations were defined as development of new symptoms, or worsening of pre-existing ones, confirmed on neurological examination and lasting at least $24 \mathrm{~h}$, in the absence of fever, preceded by stability or improvement lasting at least 30 days. MS patients who were clinically stable for 6 months prior to enrollment or more, and who did not present new T2 or Gd-enhancing lesions on magnetic resonance imaging (MRI), were considered to be in remission. No patients had received steroids or immunosuppressant treatment for at least 6 months prior to study entry. Thirty-nine patients $(85 \%)$ in remission, and $20(82 \%)$ experiencing exacerbation were on immunomodulatory treatment (interferon $\beta$ 1a) at study entry. The remaining RRMS $(n=7)$ and RREMS $(n=5)$ patients did not receive immunomodulatory or immunosuppressive treatment. None of the PPMS patients received specific immunosuppressive treatment.

Thirty healthy age- and gender-matched individuals served as controls (HCs). Underlying conditions were ruled after thorough clinical and neurological examination, as well as standard blood biochemistry tests.

Fifteen relapsing remitting MS patients presenting acute exacerbations (10 women and 5 men; mean age $34.8 \pm 6.3$ ) were followed for $37.3 \pm 3.3$ months. Every 3 months, patients underwent complete physical exam including disease activity and Expanded Disability Status Scale Score (EDSS) assessment. Brain MRI was performed at 6-months intervals on a 1.5-T Signa unit (General Electric) and axial slices, $5 \mathrm{~mm}$ thick, were obtained with T2-weighted, proton density, fast spin echo, fluid-attenuated inversion recovery, and T1-weighted sequences, before and after administration of gadolinium (Gd) diethylenetriamine pentaacetic acid $0.1 \mathrm{mmol} / \mathrm{kg}$. Results are reported as combined unique activity (CUA), calculated by combining new or enlarging T2 lesions with gadolinium-enhancing lesions as previously reported elsewhere (29).

Heparinized peripheral blood samples were drawn at time of MRI to study MAIT cells. During relapses, peripheral blood was drawn within 3-5 days of the onset of the symptoms. When peripheral blood was drawn from patients with PPMS, no contrast-enhanced lesions were evidenced in any of them. 
TABLE 1 | Demographic and clinical characteristics of the study population ${ }^{a}$.

\begin{tabular}{lcccc}
\hline Characteristics & RRRMS & RREMS & PPMS & Controls \\
\hline$n$ & 46 & 25 & 15 & 30 \\
Age (years) & $33.5 \pm 6.8$ & $35.3 \pm 7.1$ & $47.5 \pm 3.5$ & $37.3 \pm 8.9$ \\
Female:male & $30: 16$ & $16: 8$ & $8: 7$ & $20: 10$ \\
EDSS & $2.1 \pm 1.7$ & $2.3 \pm 1.8$ & $4.6 \pm 2.4$ & - \\
Disease duration & $3.8 \pm 4.3$ & $4.1 \pm 5.1$ & $4.3 \pm 4.2$ & - \\
(years) & & & & - \\
Patients under & $39(85 \%)$ & $20(82 \%)$ & - & \\
IFN- $\beta$ 1a treatment & & & &
\end{tabular}

a Values are expressed as mean $\pm S D$. IFN, interferon; RRRMS, relapsing remitting MS during remission; RREMS, relapsing remitting MS during exacerbations; PPMS, primary progressive MS; EDSS, Expanded Disability Status Scale Score.

Therefore, the presence of radiological exacerbations could not be considered.

Patients who experienced exacerbation were treated with methylprednisolone $1 \mathrm{~g}$ /day for 5 days. In these patients, peripheral blood samples were obtained before steroids treatment. Paired samples [blood and cerebrospinal fluid (CSF)] were obtained in 12 patients during exacerbations (eight women and four men; mean age $35.7 \pm 7.1$ years). CSF samples were obtained only as part of diagnostic screening in all patients. CSF samples from 12 individuals (eight women and four men; mean age $32.5 \pm 6.9$ years) undergoing extraspinal orthopedic surgery, obtained prior to spinal anesthetic injection, served as controls. Demographic and clinical characteristics of patients and controls are summarized in Table $\mathbf{1}$.

The IRB of the Dr. Raúl Carrea Institute for Neurological Research, FLENI, approved this study, and written informed consent was obtained from all participants.

\section{Flow Cytometry}

Peripheral blood mononuclear cells (PBMCs) were isolated by density-gradient centrifugation over Ficoll-PaquePLUS (Amersham Biosciences, Piscataway, NJ), and cell surface molecule labeling was performed, using appropriate monoclonal antibody (mAb) combinations including CD4-V500 (RPA-T4), CD8-V450 (RPA-T8), CCR5-PE (2D7), CD162-PE (KPL-1), CD69-PerCP-Cy5.5 (FN50), CD25-FITC (M-A251), CD58-PE (L306.4), VLA-4-PE (9F10), CD19-PE (HIB19), CD218a-PE (H44), and CD45RA-FITC (L48) from BD Biosciences (San Jose, CA); CD3-Alexa 700 (UCTH), TCR Va7.2-FITC (3C10), TCR Va7.2-PE-Cy7 (3C10), CD161-PE (HP-3G10), CD161-APC (HP-3G10), TCR- $\gamma / \delta$-FITC (B1), and TCR- $\gamma / \delta$-APC (B1) from Biolegend (San Diego, CA); CCR6-PE (53103) and CXCR6-PE (56811) from R\&D Systems (Minneapolis, MN); and CD11a-PE (ab27351) from abcam (Cambridge, MA). Isotype control mAbs were purchased from R\&D Systems. Samples were acquired with an LSRII flow cytometer (BD Biosciences) using BD FACSDiva $^{\mathrm{TM}}$ software, and analyzed with FlowJo software (Tree Star, Ashland, OR).

The size of a population (for example, MAIT cells) was reported as a percentage relative to the overall number of cells acquired in the sample under study. On the other hand, when the entire populations stain with different levels of antibody, reflecting different levels of expression of certain molecules (e.g., adhesion molecules and chemokine receptors), values were reported as MFI, since the distribution of our populations was not bimodal, and the percentages of the coefficient of variation $(\mathrm{CV})$ were $<7 \%$ in all cases.

\section{Identification of MAIT Cells}

MAIT cells were identified phenotypically as $\mathrm{CD} 3^{+} \mathrm{CD} 4^{-} \mathrm{TCR} \gamma / \delta^{-}$TRAV $1-2^{+} \mathrm{CD} 161^{\text {high }}$ by flow cytometry as previously described (Figure 1A) (10). To confirm that all MAIT cells contained the appropriate cell rearrangements, TCR genotyping in sorted $\mathrm{CD}^{+} \mathrm{TCR} \gamma / \delta^{-}$TRAV $1-2^{+}$ CD161 $1^{\text {high }}$ cells was performed. TCR $\alpha$-chain sequences spanning CDR3 region (V-J joining region) were amplified using TRAV1-2 (5'-GTCGGTCTAAAGGGTACAGT-3') and C $\alpha$ (5'-TTTAGAGTCTCTCAGCTGGTA-3') primers. Genotyping of MAIT cells from seven different randomly selected RRMS patients and seven healthy control subjects showed that isolated cells had the TRAV1-2-TRJ33 invariant sequences in the CDR3 region, confirming study cells reported to be MAIT cells were in fact MAIT cells.

For CSF immune phenotyping, $8-10 \mathrm{ml}$ of spinal fluid was obtained by lumbar puncture. Only red blood cellfree samples were studied. Because spinal fluid samples are frequently paucicellular with a rapidly decreasing cell viability, CSF was collected in tubes containing stabilization medium (RPMI-1640, with 25 mM HEPES, 1 mM glutamine, 2\% penicillin/streptomycin, $5 \%$ heat-inactivated fetal bovine serum, and 2,500 IU heparin) in all cases to prevent cell lysis (30). The maximum time elapsed between sampling and data acquisition in the flow cytometer was $3 \mathrm{~h}$. After collection, cells were concentrated by centrifugation $(8 \mathrm{~min}, 500 \mathrm{~g}$ ). Supernantants were aspirated and cells were resuspended in 100-300 $\mu \mathrm{l}$ of PBS. Next, the samples were incubated for $20^{\prime}$ on ice in the dark with each properly titrated $\mathrm{mAb}$, and after incubation, the cells were washed in PBS. For absolute cell number calculation, $100 \mu \mathrm{l}$ of PBS and $100 \mu \mathrm{l}$ of CytoCount counting beads (Dako, Glustrop, Denmark) were added. Data were acquired and analyzed immediately after sample preparation was completed. Absolute cell numbers were calculated using the following formula:

(events in cell subset gate/events in counting bead gate) $\times$ (counting beads added per tube/volume of CSF sample $)=$ cells/ $\mu 1(30)$.

\section{Quantification of Secreted Cytokines by MAIT Cells}

For in vitro studies, MAIT cells $\left(\mathrm{CD}^{+}{ }^{+} \mathrm{CD} 4^{-} \mathrm{TCR} \gamma / \delta^{-} \mathrm{TRAV} 1-\right.$ $\left.2^{+} \mathrm{CD} 161^{\text {high }}\right)$ were isolated from PBMCs using a FACSAria ${ }^{\mathrm{TM}}$ III cell sorter (BD Biosciences). Although MR1-antigen tetramer staining has been suggested by some authors as the best method for MAIT cell isolation, other studies have demonstrated that not all MR1-antigen tetramer ${ }^{+}$cells are MAIT cells $(14,31)$.

To analyze cytokine production, MAIT cells were cultured in 96-well flat-bottom plates at $1 \times 10^{5}-3 \times 10^{4}$ cells per well 


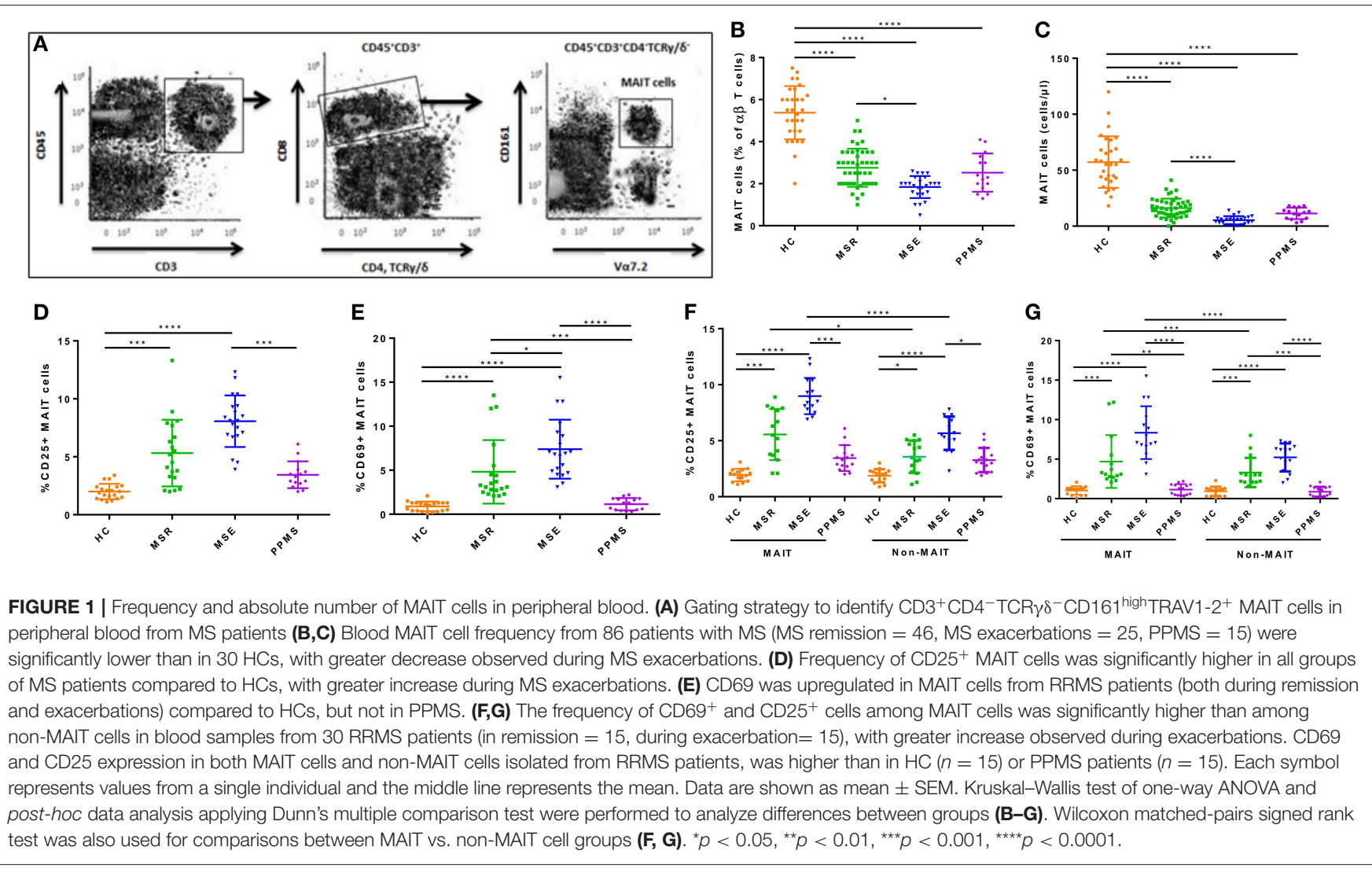

with complete RPMI-1640 media supplemented with $10 \%$ heatinactivated fetal bovine serum (FBS; Life Technologies BRL, Grand Island, NY). For mitogen stimulation, MAIT cells were stimulated with a combination of phorbol 12-myristate 13acetate (PMA; $100 \mathrm{ng} / \mathrm{ml})$ and ionomycin $(1 \mu \mathrm{g} / \mathrm{ml}$; both from Sigma-Aldrich) for $48 \mathrm{~h}$. IFN- $\gamma$, IL-4, IL-17, GM-CSF, and TNF$\alpha$ secretion was measured by single-cell resolution enzymelinked immunospot (ELISPOT) assay, as described previously (32), using commercially available ELISPOT kits, following manufacturer instructions. IFN- $\gamma$, IL-4, IL-17, and TNF- $\alpha$ ELIPSOT detection kits were purchased from R\&D Systems, and the GM-CSF ELISPOT detection kit was purchased from Active Bioscience (Hamburg, Germany). Cytokine secretion was measured during MR1-dependent stimulation, for which $\mathrm{HeLa}$ cells (American Type Culture Collections, ATCC, Manassas VA) were transfected with MR1 and untransfected HeLa cells were cultured in Eagle's Minimum Essential Media (Sigma-Aldrich), together with Escherichia coli (strain D21; ATCC) fixed in 1\% paraformaldehyde for $3 \mathrm{~min}$ at infectivity levels increased in multiples of 10 (33). After $3 \mathrm{~h}$, HeLa cells were washed and incubated an additional hour with Eagle's Minimum Essential Media supplemented with $10 \%$ FBS and $50 \mu \mathrm{g} / \mathrm{ml}$ gentamicin (Sigma-Aldrich). Infected HeLa cells $\left(1 \times 10^{5}\right.$ per well) used as antigen-presenting cells (APCs) were cultured with isolated MAIT cells $\left(1 \times 10^{5}\right.$ cells per well $)$ for $48 \mathrm{~h}$ in complete media supplemented with $10 \%$ FBS. Cytokine secretion was measured using ELISPOT. For blocking experiments, anti-MR1 (26.5), anti
Class-I HLA-ABC (W6/32), or IgG2 isotype control (all from Biolegend) were added $60 \mathrm{~min}$ before stimulation at $10 \mu \mathrm{g} / \mathrm{ml}$ final concentration.

In some experiments, MAIT cell clones were tested for cytokine production 7 to 10 days after their most recent stimulation. Cloned MAIT cells were stimulated as previously described, and after 36 (for IL-4) or $72 \mathrm{~h}$ (for the other cytokines), supernatants were harvested and stored at $-70^{\circ} \mathrm{C}$ for later tests. Concentrations of IFN- $\gamma$, IL-4, IL-17, GM-CSF, and TNF- $\alpha$ in culture supernatants were determined using commercially available ELISA kits (R\&D Systems), following manufacturer instructions.

In all cases, the optimal time courses of each cytokine production by cloned and uncloned MAIT cells were established in preliminary experiments and were defined as the maximum cytokine production obtained after each experimental condition.

\section{Cloning of MAIT Cells}

$\mathrm{CD} 3^{+} \mathrm{CD} 4^{-} \mathrm{TCR} \gamma / \delta^{-}$TRAV $1-2^{+} \mathrm{CD} 161^{\text {high }}$ cells were sorted from PBMCs as previously described and cloned using limiting dilution (9). Purity of the cell sorting was $>96 \%$. Cells were stimulated with phytohemagglutinin (PHA, Sigma-Aldrich; $1 \mu \mathrm{g} / \mathrm{ml}$ ), and $100 \mathrm{U} / \mathrm{ml}$ recombinant human IL-2 (rhIL-2; R\&D Systems), in the presence of $1 \times 10^{5}$ autologous irradiated PBMCs. $T$ cell clones were re-stimulated weekly following the same protocol. To obtain CSF MAIT cell clones, fresh CSFderived mononuclear cells were isolated after CSF centrifugation, 
resuspended in complete culture media, and expanded using autologous irradiated PBMCs $\left(5 \times 10^{4} /\right.$ well $)$ plus PHA. Cells were re-stimulated with rhIL-2 every 7 days, for 3 weeks. After CSF mononuclear cell expansion, MAIT cells were isolated and cloned by limiting dilution as previously described.

\section{MAIT Cell Clone TCR Sequencing}

TCR $\alpha$ and TCR $\beta$ chain high-throughput deep RNA sequencing was performed as previously described (9). Briefly, RNA from MAIT cell clones obtained from peripheral blood and CSF was prepared using TRIZOL (Invitrogen, Carlsbad, CA) following manufacturer instructions, and cDNA was synthesized using an Illumina TruSeq RNA sample preparation kit (San Diego, CA), with multiplexing indexes. Purified cDNA libraries were subjected to an indexed Paired-End sequencing run of $2 \times 51$ cycles on an Illumina HSeq 2000 platform. For CDR3 $\beta$ region analysis, a multiplex PCR system was used to amplify CDR3 $\beta$ sequences from cDNA samples using 52 forward primers for the $\mathrm{V} \beta$ gene segment, and 13 reverse primers for the $\mathrm{J} \beta$ segment. Using this method, it is possible to obtain a 60-bp fragment capable of identifying the VDJ region encompassing each unique CDR3 $\beta$ (34). Amplicons were sequenced using the Illumina HiSeq platform. Artificially added sequences (Tag, adaptors), and sequences with low-quality scores were removed using a modified nearest-neighbor algorithm. Remaining sequences were used for assignment of TCR $\alpha \mathrm{V}, \mathrm{J} \alpha, \operatorname{TCR} \beta \mathrm{V}, \mathrm{D}$, and $\mathrm{J} \beta$ genes, based on definitions provided by the International Immunogenetics Information (IMGT, www.imgt.org) database, and results were analyzed using the ImmunoSEQ analyzer toolset.

\section{Statistical Analysis}

Statistical analyses were performed using SPSS v 22 and Prism 6 (GraphPad) software. Kolmogorov-Smirnov test was used to evaluate distribution of variables and Mann-Whitney or Student's $t$-test was performed to compare continuous data between groups as appropriate. Kruskal-Wallis of oneway ANOVA, a nonparametric test, was used to estimate differences between more than two groups and post-hoc analysis was conducted using Dunn's multiple comparison test, when appropriate. Wilcoxon-Mann-Whitney test was used to assess whether the treatment had an impact on MAIT cell percentages during both remission and relapse. Wilcoxon matched-pairs signed-rank test was used for comparison between two paired groups. In addition, Spearman's correlation was used to analyze associations between frequency of MAIT cells and new or enlarging T2 lesions, Gd+ lesions, and CUA on brain MRI. The optimal diagnostic cutpoint between the number of MAIT cells and relapses was estimated using a ROC curve with the nearest method (cutpt command in Stata v12.1). For all tests, $p<0.05$ were considered statistically significant.

\section{RESULTS}

\section{MAIT Cells Are Reduced in Peripheral Blood of MS Patients}

Percentages and absolute numbers of MAIT cells in peripheral blood samples from the different cohorts were determined by flow cytometry. MAIT cells were defined as $\mathrm{CD}^{+} \mathrm{CD}^{-}{ }^{-} \mathrm{TCR} \gamma / \delta^{-} \mathrm{TRAV} 1-2^{+} \mathrm{CD} 161^{\text {high }}$ (Figure 1A). Because relapsing remitting and primary progressive patients differ significantly in age (Table 1), two control groups were initially evaluated, one similar in age to relapsing remitting patients $(n=20)$, and another to primary progressive ones $(n=10)$. Because no statistically significant differences were observed in percentages or absolute numbers of MAIT cells between groups ( $p=0.33$ and $p=0.45$, respectively), they were subsequently pooled into one. As illustrated in Figure 1B, percentage of MAIT cells out of the total TCR $\alpha \beta$ T cells was significantly lower in both MS patients during remission and PPMS subjects compared to HCs $[2.75 \pm 0.90$ vs. $5.37 \pm 1.26$ ( $p$ $<0.0001), 2.52 \pm 0.90$ vs. $5.37 \pm 1.26$ ( $p<0.0001$ ), respectively]. In addition, MAIT cell number decrease was even more robust during MS exacerbations than during remission ( $1.84 \pm 0.52$ vs. $2.75 \pm 0.90 ; p<0.05)$. No significant differences were observed between MS patients in remission or MS patients during exacerbations vs. PPMS ( $p=0.99$ and $p=0.48$, respectively). RRMS patients in remission or experiencing exacerbations had significantly lower absolute MAIT cell numbers compared to HCs $[16.22 \pm 8.38$ vs. $57.23 \pm 23.05$ cells/ $\mu l(p<0.0001)$, and $5.28 \pm 3.65$ vs. $57.23 \pm 23.05$ cells $/ \mu l(p<0.0001)$, respectively; Figure 1C]. MAIT cell numbers were also decreased in PPMS patients compared to HCs $[11.33 \pm 11.05$ vs. $57.23 \pm 23.05$ cells/ $\mu 1(p<0.0001)$, Figure 1C]. There were no statistically significant differences between MS patients in remission and PPMS patients $(p=0.55)$. Of note, frequency of TRAV1- $2^{+}$ CD161 $1^{\text {low }}$ and TRAV1-2 $2^{+}$CD161 ${ }^{-}$or TRAV1-2- CD161 $1^{\text {high }}$ cell populations did not differ between MS patients and HCs, suggesting that reduced MAIT cell numbers in MS patients were not due to down-modulation of TRAV1-2+ TCR or CD161 molecules in MAIT cells of MS patients.

Human MAIT cells predominantly express the CD8 $\alpha$ coreceptor $\left(\mathrm{CD}^{+}\right)$, with a smaller subset not expressing any $\mathrm{CD}^{-} \mathrm{CD}^{-}$(double negative; $\mathrm{DN}$ ), and very few $\mathrm{CD}^{+}$cells expressing the canonical TCR. Recently, significant functional differences have been described between both MAIT cell subsets, with important implications during infectious and inflammatory diseases. The findings suggest that the DN MAIT cell subset may derive from the main $\mathrm{CD}^{+}$MAIT cell subpopulation (35). We therefore decided to investigate the frequency and number of both subsets in the different study populations. As illustrated in Supplementary Figures S1A,B, both the percentage and absolute number of total MAIT cells and $\mathrm{CD} 8^{+}$MAIT cells were significantly reduced in peripheral blood samples from RRMS patients in remission and during exacerbations, as well as in PPMS subjects compared to HC $(p<0.0001)$. Likewise, both percentage and absolute number of DN MAIT cells were also lower in all three MS patient cohorts compared to HC, although the drop was of lesser magnitude $(p<0.01$ to $p<0.001)$.

Even though the number of untreated patients was small, we found no statistically significant differences in the percentage of MAIT cells between untreated [median, range $(2.5 ; 1-5)$ ] and IFN- $\beta$ 1a-treated $(3 ; 2-4.5)$ remission patients $(p=0.69)$. Likewise, there were no differences in untreated $(1.95 ; 0.5-2.6)$ and IFN- $\beta$ 1a-treated $(2 ; 1.8-2.5)$ relapsing patients $(p=0.58)$. 
These data suggest that the decrease in MAIT cells is part of the pathophysiological process of the disease and not a consequence of the treatment received. On the other hand, since none of the PPMS patients received specific treatment for MS, we can exclude an effect of specific therapies for MS on the results observed in this group of patients.

\section{MAIT Cell Characterization}

To assess whether ex vivo MAIT cells from MS patients have a different state of activation than those from HCs, we investigated CD25 and CD69 expression. Percentages of CD25 ${ }^{+}$MAIT cell numbers were significantly higher in MS patients during exacerbations than in MS patients in remission or in PPMS patients $[8.07 \pm 2.23$ vs. $5.32 \pm 2.87$ cells $/ \mu l(p<0.0001)$, and $8.07 \pm 2.23$ vs. $3.44 \pm 1.55$ ( $p<0.0001)$, respectively; Figure 1D]. CD69 was upregulated in MAIT cells from RRMS patients (both in remission and during exacerbations) compared with HCs $[4.82 \pm 3.61$ vs. $0.89 \pm 0.54(p<0.0001)$, and $7.39 \pm$ 3.34 vs. $0.89 \pm 0.54(p<0.0001)$, respectively; Figure 1D], but not in PPMS patients [1.14 \pm 0.69 vs. $0.89 \pm 0.54(p=0.81)$; Figure 1E]. No statistically significant differences were observed in CD69-expressing MAIT cell numbers between RRMS patients during exacerbations vs. remission $(p=0.13)$. Likewise, CD25 and CD69 expression, measured as mean fluorescence intensity (MFI), was significantly higher in RRMS patients than in HC and PPMS, particularly during exacerbations $(p<0.05$ and $p$ $<0.01$, respectively). These findings prompted us to investigate whether these observations were MAIT cell-specific or shared by non-MAIT memory cells (defined as $\mathrm{CD}^{+}{ }^{+} \mathrm{CD} 45 \mathrm{RA}^{-}$TRAV1$2^{+/-} \mathrm{CD} 161^{\text {low }} \mathrm{CD}^{+}$). In RRMS patients, the frequency of $\mathrm{CD} 9^{+}$and $\mathrm{CD} 25^{+}$cells was higher among MAIT cells than nonMAIT cells ( $p<0.05$ to $p<0.0001$; Figures 1 F,G). In addition, expression of both activation markers was even more robust during MS exacerbations $(p<0.0001)$. Of note, the percentage of both $\mathrm{CD}^{\circ} 9^{+}$and $\mathrm{CD} 25^{+}$cells among MAIT cells and nonMAIT cells isolated from RRMS patients in remission and during exacerbations were significantly higher than in HC and PPMS patients ( $p<0.05$ to $p<0.0001$; Figures 1F,G). Overall, these results indicate that MAIT cell number decrease in MS patients is accompanied by activated phenotype, suggesting that abnormal MAIT cell activation is present in MS patients, particularly during active disease.

Since MAIT cells can migrate to inflamed tissues (20), we next measured surface expression on ex vivo MAIT cells of selectins and integrins, chemokine receptors important for CNS invasion. CCR5, CCR6, and CXCR6 expression, measured as MFI, was significantly higher in all three MS patient cohorts than in HCs $(p<0.0001$; Figure 2A). Once again, we set out to investigate whether these observations were MAIT cell-specific, or shared by non-MAIT memory cells, and found that MAIT cells from MS patients expressed CCR5, CCR6, and CXCR6 at higher levels than non-MAIT cells, with greater expression during exacerbations ( $p$ $<0.05$ to $p<0.001$; Supplementary Figures S2A-C). Likewise, MAIT cells from MS patients showed increased MFI for different adhesion molecules and integrins, especially those involved in the first step of lymphocyte extravasation into CNS, including VLA-4 (CD49d), LFA-1 (CD11a), PSGL-1 (CD162), and LFA-3
(CD58), compared to HCs ( $p<0.05$ to $p<0.0001$; Figure 2B). Expression levels of the molecules analyzed did not differ between MS patients in remission or during exacerbations (Figure 2B). Next, we investigated surface expression of adhesion molecules and integrins on MAIT cells and non-MAIT cells, in the same cohort in which we had previously quantified chemokine receptor levels. In MS patients, expression of VLA4 was significantly higher in MAIT cells than in non-MAIT cells, with greatest expression occurring during exacerbations ( $p$ $=0.01$ to $p<0.001$; Supplementary Figure S2D), whereas no significant difference was observed between cell populations in LFA-1, PSGL-1, and LFA-3 surface expression ( $p=0.33$ to $p=$ 0.69; Supplementary Figures S2E-G). These specific phenotypic characteristics on MAIT cells from RRMS patients (namely, higher levels of CCR5, CCR6, CXCR6, and VLA-4 expression) suggest that MAIT cells are more prone to migrate toward the inflamed CNS.

To further explore MAIT function in MS, we analyzed cell cytokine production. To this end, isolated MAIT cells were stimulated with a combination of PMA plus IM. As shown in Figure 3A, RRMS patients generated significantly higher numbers of IFN- $\gamma$, IL-17, GM-CSF, and TNF $\alpha$-producing MAIT cells $(p<0.001$ to $p<0.0001)$ both during remission and exacerbation than HCs. In contrast, MAIT cell numbers producing any of these cytokines did not differ between PPMS and HCs, while IL-4-producing MAIT cell numbers were similar in all four study cohorts. Similar results were observed when MAIT cells were stimulated in an MR1-dependent manner ( $p$ $<0.05$ to $p<0.0001$; Figure 3B). To confirm these responses, MAIT cells were isolated ex vivo from 10 RRMS patients in remission and were stimulated with HeLa MR1 transfected HeLa cells infected with fixed E. coli, in the presence and in the absence of an anti-MR1 blocking antibody. As shown in Figures 3C,D, activation was severely inhibited after addition of the blocking antibody, but not after addition of an MHC Class I blocking antibody (HLA-ABC) or of a control isotype. Taken together, these results suggest that this response is MR1-dependent.

Given the fact that the IL-18 receptor is expressed on MAIT cells (36), and that serum IL-18 is known to be increased in MS patients (23), as well as present in MS CNS lesions (23-37), we set out to investigate whether MAIT cells were activated by IL-18. As shown in Figures 3E-H, MAIT cells isolated ex vivo from RRMS patients both in remission and during exacerbations responded robustly to IL18-driven stimulation with significantly higher expression of CD69, VLA-4, and production of IL-17, compared to PPMS patients and HCs $(p<0.01$ to $p<0.0001)$. These effects were significantly reduced in the presence of the p38-MAPK inhibitor SB203580, which suppresses IL-18 signaling (38). Overall, these results indicate that MAIT cells can be activated by IL-18 through a TCR-independent mechanism. As previously demonstrated, IFN- $\gamma$ secretion, however, was not induced by isolated IL-18 stimulation (23), requiring addition of IL-12. Addition of IL-12 to culture medium significantly increased IFN- $\gamma$ production in RRMS patients both during exacerbation and while in remission, compared to PPMS patients and HC $(p<0.0001)$. 

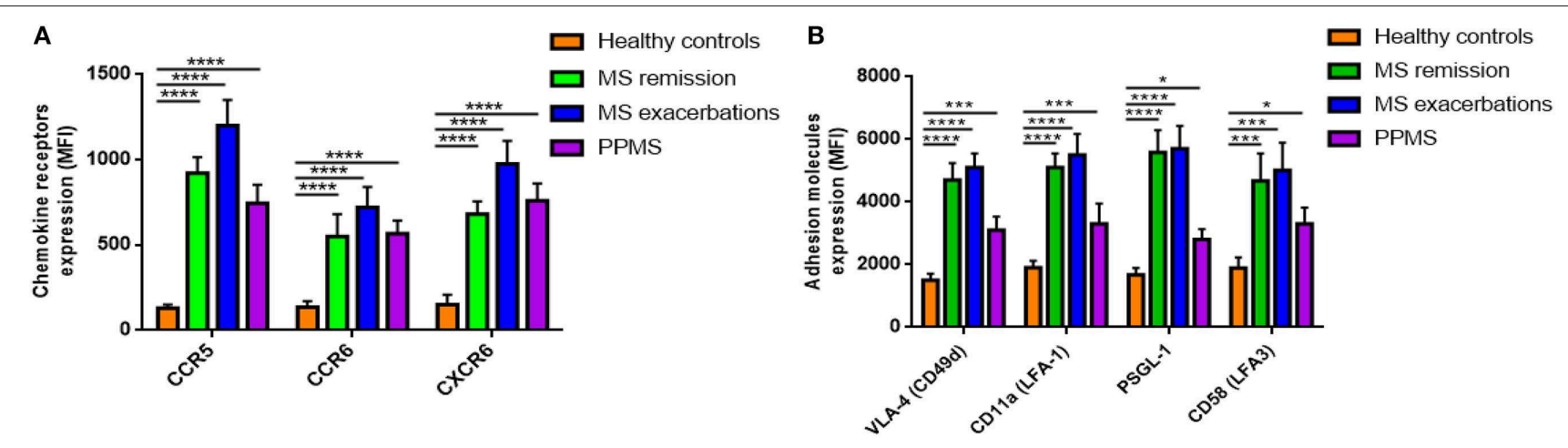

FIGURE 2 | Expression of chemokine receptors and adhesion molecules. (A) Expression of CCR5, CCR6, and CXCR6, and (B) the adhesion molecules VLA-4 (CD49d), LFA-1 (CD11a), PSGL-1 (CD162), and LFA-3 (CD58) was measured by flow cytometry and results are expressed as mean fluorescence intensity (MFI). MAIT cell values were significantly higher in MS patients than in HCs. Data are presented as mean values \pm SEM from 40 RRRMS patients, 25 RREMS patients, 15 PPMS patients, and 25 healthy control subjects. Statistical analysis was performed using the Wilcoxon matched-pairs signed rank test. ${ }^{\star} p<0.05$, ${ }^{\star \star \star} p<0.001,{ }^{\star \star \star \star} p<$ 0.0001.

\section{Variations in MAIT Cell Numbers During MS Course}

To further explore the link between MAIT cells and clinical characteristics, we analyzed MAIT cell levels in 15 RRMS patients followed for at least 36 months, as well as MRI results at 3and 6-months intervals, after an exacerbation. As shown in Figure 4A, MAIT cell levels were lower at the time of MS relapse. Three months later, MAIT cell percentages increased significantly along with the clinical recovery, persisting at high values while disease remained stable. Interestingly, seven patients (Figure 4B) showed a further decline in MAIT cell percentages at $6,18,24$, and 30 months (patient numbers 1, 3, 7, 9, 10, 11, and 14) follow-up, coinciding with new or enlarging T2 lesion and/or Gd enhancing lesions on brain MRI. None of the patients presented clinical relapses during this follow-up period. Thus, an inverse correlation between MAIT cell numbers and either new or enlarging T2 lesions $(r=-0.69, p<$ 0.0001), Gd-enhancing lesions ( $r=-0.72, p<0.0001)$, or CUA score $(r=-0.82, p<0.0001)$ was observed (Figure 4B and Supplementary Figure S3). In contrast, patients in whom MAIT cell percentage remained elevated during follow-up showed no evidence of MRI activity (patients 6 and 8 ). These results suggest that peripheral blood MAIT cell levels may reflect MS activity. Application of a predictive model defining disease activity as a function of MAIT cell numbers demonstrated a cutoff point of 1.7 , with 0.99 sensitivity, 0.95 specificity, and 0.97 AUC (Figure 5A). To examine whether decrease in circulating MAIT cells in MS might be linked to increased cell recruitment into the CNS, paired samples of peripheral blood and CSF were obtained from 12 RRMS patients during exacerbations, 12 RRMS patients in remission, and 12 healthy controls. MAIT cell numbers were calculated using flow cytometry. As illustrated in Figure 5B, MAIT cell frequency was significantly higher in CSF of RRMS patients during exacerbations than in peripheral blood (mean frequency $5.8 \pm 0.6$ vs. $2.3 \pm 0.2 \% ; p=0.0005$ ). By contrast, no difference was observed in MAIT cell percentages between peripheral blood and CSF during remission (mean frequency 4.4 $\pm 0.2 \%$ vs. $3.9 \pm 0.3 \%$; $p=0.09$; Figure 5 B). MAIT cell frequency in CSF from healthy controls was statistically significantly lower compared to MS patients during remission $(p=0.01)$ and exacerbation $(p=0.0005)$. Of note, non-MAIT cells were also found to be slightly increased in CSF compared to peripheral blood during MS exacerbations, but not during remissions ( $p=$ 0.44 , and $p=0.49$, respectively; Figure 5C). Further analysis of $\mathrm{HC}$ revealed higher numbers of MAIT cells in peripheral blood compared to CSF (mean frequency $5.8 \pm 0.2 \%$ vs. $2.1 \pm 0.2 \%$; $p=0.0005$; Figure 5D). Taken together, these observations are consistent with a preferential recruitment of MAIT cells into the CNS during acute exacerbations.

\section{MAIT Cell T-Cell Receptor Repertoire}

Fifteen MS patients were followed over time for a period of at least 3 years. In this cohort, we were able to isolate 34 MAIT cell clones from peripheral blood in seven patients at study entry. In four of these patients, CSF-sorted MAIT cells (yield $=0.5-0.9$ cells $/ \mu \mathrm{l}$ ) were expanded as described in Methods and then cloned by limiting dilutions. Using this method, a total of 23 MAIT cell clones were isolated from CSF. Ninety percent of peripheral blood MAIT cell clones expressed the canonical $\alpha$ chain TRAV12 -TRJ33 transcript. However, TCR sequence analysis showed that some MAIT cell clones transcribed a functional TRAV1-2-TRJ12 (6\%) or TRAV1-2-TRJ20 (4\%) rearrangement.

All clones were $\mathrm{CD}^{-}$and $\mathrm{CD} 161^{+}$. Twenty-five clones expressed the CD8 molecule, whereas nine clones were $\mathrm{CD} 4^{-}$ $\mathrm{CD}^{-}$(S1 Table).

Analysis of TCRV $\beta$ sequences in all 34 MAIT cell clones isolated from peripheral blood revealed that they represented a restricted number of $\mathrm{CDR} 3 \beta$ amino acid clonotypes. As previously reported $(7,9)$, strong bias of TCRV $\beta$ usage was observed: TRBV6-1, TRBV6-4, TRBV6-5, and TRBV20-1 families combined accounted for $79 \%$ of total MAIT repertoire (S1 Table and Figure 6A).

To study whether repertoire biases present in peripheral blood of MS patients reflected bias in the CNS, we compared TCRV $\beta$ 


\section{A}

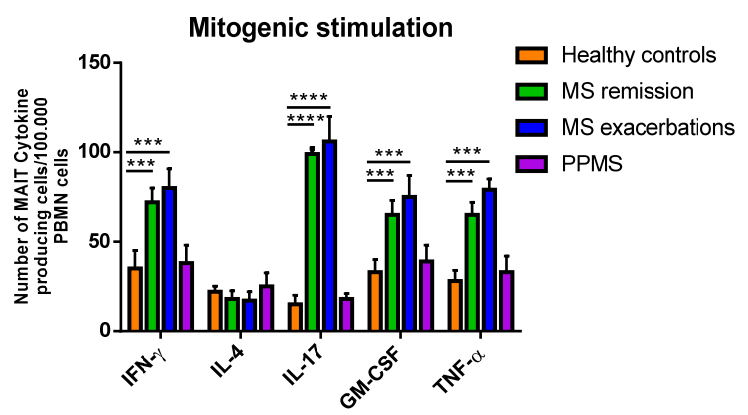

C

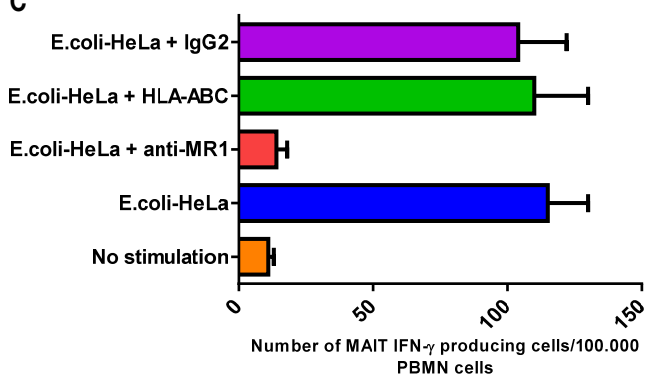

E

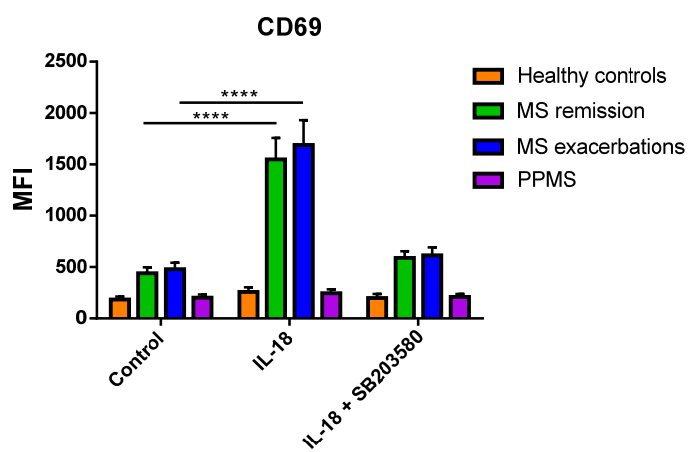

G

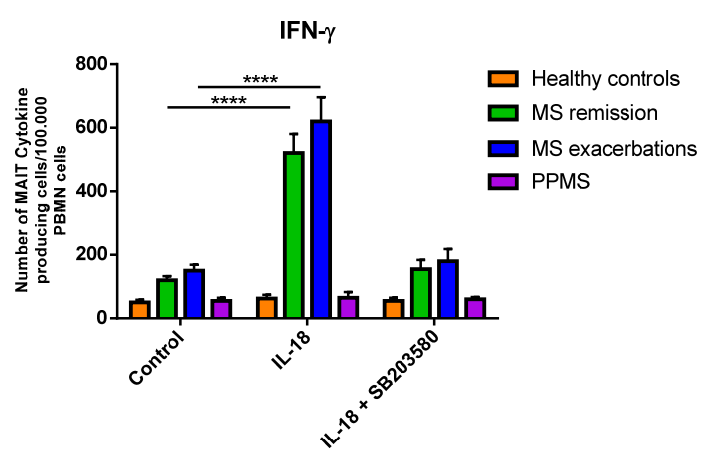

B

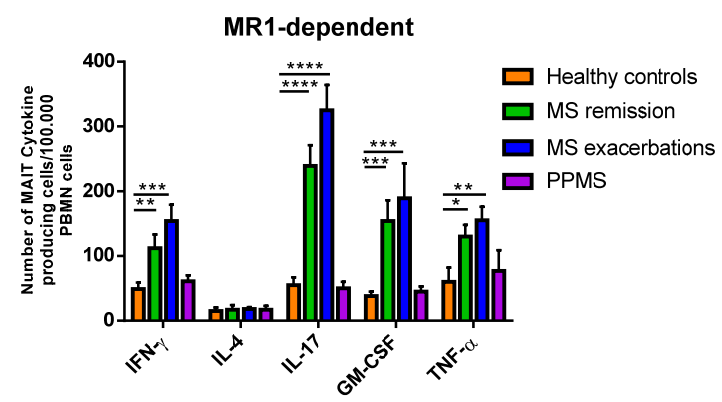

D

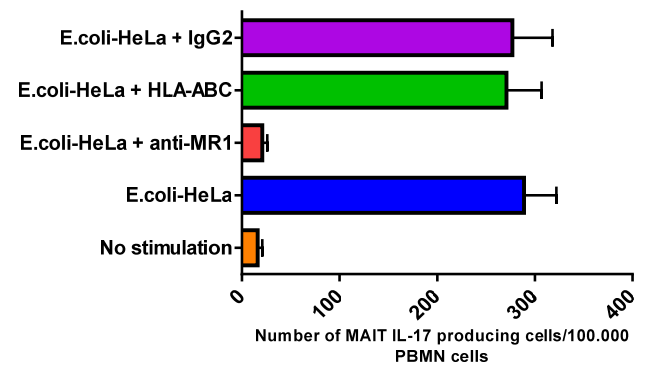

$\mathbf{F}$

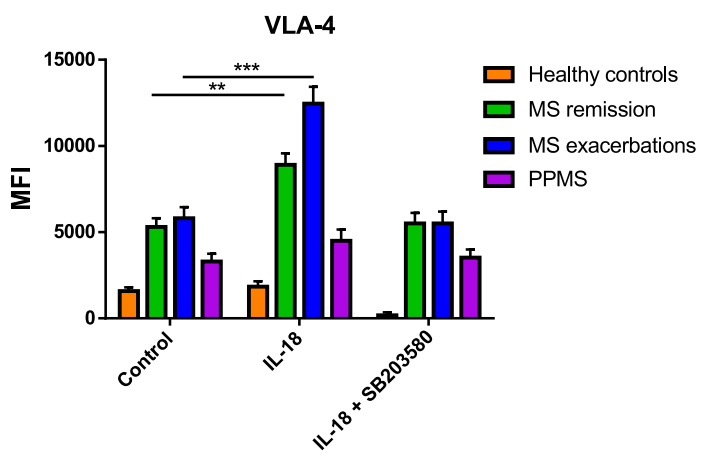

H

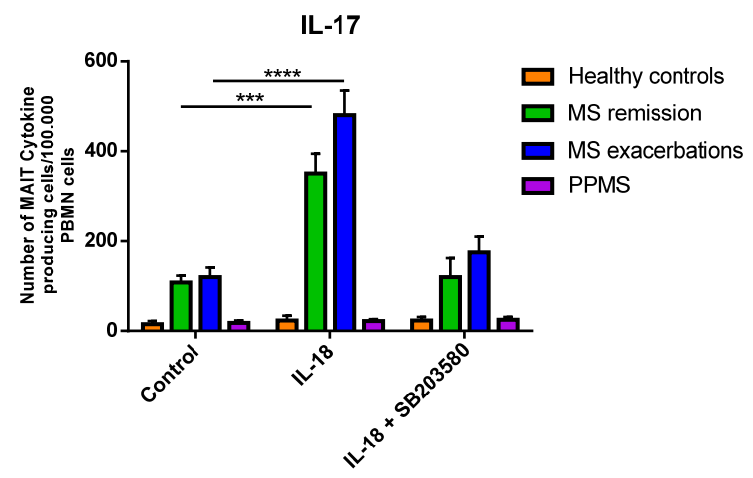

FIGURE 3 | MAIT cells produce pro-inflammatory cytokines after activation. (A) For mitogenic stimulation, sorted MAIT cells were stimulated in vitro with PMA plus ionomycin, and cytokine secretion was measured using ELISPOT assay. RRMS patients (both in remission and during exacerbation) showed significantly higher numbers of IFN- $\gamma$, IL-17, GM-CSF, and TNF $\alpha$ MAIT-producing cells compared to HCs and PPMS patients. In contrast, PPMS and HCs showed similar values of the four cytokines. The number of IL-4 MAIT-producing cells was similar in all four cohorts. (B) Similar results were observed when MAIT cells were stimulated in an MR1-dependent manner, using HeLa MR1 transfected cells infected with fixed E. coli. Data are presented as mean values \pm SEM from 40 RRRMS patients, 25 
FIGURE 3 | RREMS patients, 15 PPMS patients, and 25 healthy control subjects. (C,D) The response was MAIT cell-specific, since it was blocked by an anti-MR1 monoclonal antibody. Data are presented as mean values \pm SEM from 10 RRMS patients in remission. (E,F) For TCR-independent activation, ex vivo MAIT cells were stimulated with IL-18 $(50 \mathrm{ng} / \mathrm{ml})$, in the presence and in the absence of the p38-MAPK inhibitor SB203580 (5 $\mu \mathrm{M}$; Sigma-Aldrich), and expression of CD69 and VLA-4 was assessed using flow cytometry. Results are presented as mean values \pm SEM of mean fluorescence intensity (MFI) from 15 RRMS patients in remission, 15 RRMS patients during exacerbation, 15 PPMS patients, and 15 healthy control subjects. (G) Likewise, ex vivo isolated MAIT cells were stimulated using a similar protocol and IFN- $\gamma$ and IL-17 secretion measured using ELISPOT assay. (H) Increase in IFN- $\gamma$-secreting cell numbers was observed only after adding IL-12 (10 ng/ml) to cultures. Cytokine-secreting cell numbers were calculated by subtracting the number of spots obtained in the absence of stimulation (background control cultures), from the number of spots obtained in cultures exposed to the different stimuli. Data correspond to number of spots per $10^{5}$ PBMCs. Data are presented as mean values \pm SEM from seven different experiments. Statistical analysis was performed using the Wilcoxon matched-pairs signed rank test. ${ }^{*} p<0.05,{ }^{* *} p<0.01,{ }^{* \star *} p<$ $0.001,{ }^{* \star \star \star} p<0.0001$

repertoire from paired samples of peripheral blood and CSF. Twenty-three MAIT cell clones isolated from CSF revealed CDR3 clonotype distribution comparable to that observed in peripheral blood MAIT cell clones. TRBV6-1, TRBV6-4, and TRBV201 families combined accounted for $78 \%$ of the total MAIT repertoire (Figure 6B and S2 Table). These results suggest that oligoclonality is a shared feature of both CSF and circulating MAIT cells (Figure 6C). Furthermore, most MAIT cell clones (82\%) expressed high levels of CD103, a defining marker of tissue residency, and on activation, most produced IFN- $\gamma$ (43\%) or IL-17 (39\%; S2 Table), suggesting that resident MAIT cells are pro-inflammatory, causing deleterious effects on disease.

To determine whether sorting and cloning strategies used during isolation of CSF and blood MAIT cell clones induced a bias in the repertoire observed, by preferentially expanding certain clonotypes, MAIT cell clones derived from peripheral blood and CSF from 12 healthy controls were also studied. Although the cloning efficiency observed in peripheral blood from RRMS patients and healthy controls was similar, the scarce number of cells present in the CSF of control individuals significantly reduced the cloning efficiency compared to that observed in CSF from RRMS patients ( $<1 \%$ vs. $3-5 \%)$. Only in 3 out of $12 \mathrm{HCs}$ were we able to obtain 7 MAIT cell clones. In the same subjects, 16 MAIT cell clones were isolated from peripheral blood. Analysis of CDR3 $\beta$ amino acid sequences in peripheral blood showed a similar repertoire to that of RRMS patients, with TRBV6-1, TRBV6-4, TRBV6-5, and TRBV20-1 families combined accounting for $69 \%$ of total MAIT repertoire ( $\mathbf{S 3}$ Table). Taken together, these findings indicate that no bias was introduced by the cloning strategies used. MAIT cell clones isolated from CSF showed a preferential clonotype distribution represented by TRBV6-1, TRBV6-4, and TRBV6-5, which accounted for $71 \%$ of the total MAIT cell repertoire (S3 Table). However, when the oligoclonality observed in peripheral blood and CSF of healthy subjects was analyzed individually, contrary to what was found in RRMS patients, CDR3 clonotypes observed in paired samples of peripheral blood and CSF differed significantly in individual subjects (S3 Table). These results, however, should be interpreted with caution given the low number of MAIT cell clones isolated from CSF in healthy individuals. Next, to explore whether clonotypic characteristics translated into differential functional capacity in MAIT cell clones isolated from peripheral blood and CSF, the clones were stimulated with PMA/ionomycin and subsequent cytokine (IFN$\gamma$, IL-4, IL-17, GM-CSF, and TNF- $\alpha$ ) production was assessed. Only in 7 of the 16 MAIT cell clones isolated from peripheral blood was it possible to detect IL-17, IFN- $\gamma$, or GM-CSF production. In the case of MAIT cell clones isolated from CSF, only two of the seven clones identified were able to produce IL-17 or IFN- $\gamma$ (S3 Table). Similar results were observed after E. coli stimulation.

Most MAIT cell characterization was first carried out in blood samples. The isolation of several MAIT cell clones from paired samples of CSF and peripheral blood from four RRMS patients provided a unique opportunity to study and compare their functional response. To investigate whether MAIT cell clones isolated from these two different sources presented different functional capacities, each was stimulated with either E. coliinfected HeLa cells or with PMA/ionomycin. Production of IL-17 and IFN- $\gamma$ in the supernatants was then assessed using ELISA. As illustrated in Figure 7A, MAIT cell clones isolated from CSF secreted significantly higher amounts of IL-17 and IFN- $\gamma$ after PMA/ionomycin stimulation, compared to MAIT cell clones isolated from peripheral blood $[520.0 \pm 59.4 \mathrm{pg} / \mathrm{ml}$ vs. $1060.0 \pm$ $129.9 \mathrm{pg} / \mathrm{ml}(p<0.0001)$ and $1425.0 \pm 149.8 \mathrm{pg} / \mathrm{ml}$ vs. 3675.0 $\pm 311.2 \mathrm{pg} / \mathrm{ml}(p<0.0001)$, respectively]. In a second protocol, MAIT cell clones from both CSF and peripheral blood stimulated with E. coli-infected HeLa cells also secreted significantly higher amounts of IL-17 and IFN- $\gamma$ compared to those from peripheral blood $[720.4 \pm 92.6 \mathrm{pg} / \mathrm{ml}$ vs. $1950.5 \pm 234.1 \mathrm{pg} / \mathrm{ml}(p=0.0003)$ and $3200.0 \pm 379.4 \mathrm{pg} / \mathrm{ml}$ vs. $6965.0 \pm 708.0 \mathrm{pg} / \mathrm{ml}(p<0.001)$, respectively; Figure $7 \mathbf{B}$ ]. Furthermore, stimulation with different concentrations of IL-18 revealed that CSF MAIT cell clones were more sensitive to lower IL-18 concentrations compared to peripheral blood MAIT cell clones in terms of both IL-17 and IFN- $\gamma$ production ( $p=0.03$ and $p=0.01$; Figures 7 C,D). Indeed, stimulation with IL-18 of CSF MAIT cell clones induced $50 \%$ of the maximum secretion of IL-17 at a concentration of $3.5 \mathrm{ng} / \mathrm{ml}$, whereas in peripheral blood MAIT cell clones, a concentration of $23 \mathrm{ng} / \mathrm{ml}$ was necessary to achieve a similar effect (6.5 times greater; Figure 7C). Likewise, stimulation of CSF MAIT cell clones with $4 \mathrm{ng} / \mathrm{ml}$ of IL-18 induced $50 \%$ of the maximum IFN- $\gamma$ secretion, whereas $25 \mathrm{ng} / \mathrm{ml}$ were required in peripheral blood MAIT cell clones (6.25 times more; Figure 7D). Due to the restricted number of cells in CSF, it was not possible to investigate other functional responses. Taken together, these results indicate that CSF MAIT cells respond more strongly in terms of pro-inflammatory cytokine secretion compared to peripheral blood MAIT cells, suggesting that these cells exert a potentially harmful role in MS. Alternatively, the altered cytokine pattern may be due to a different milieu in CSF as compared to peripheral blood. 


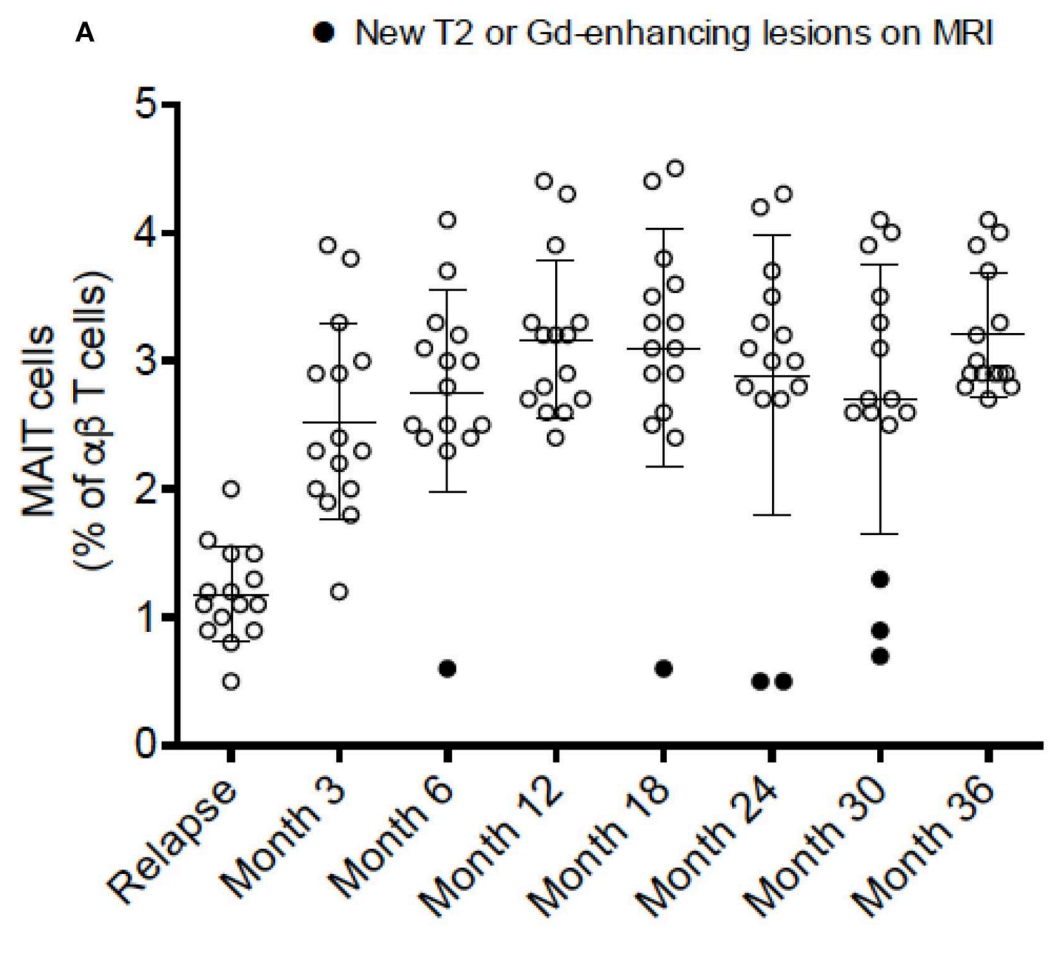

B
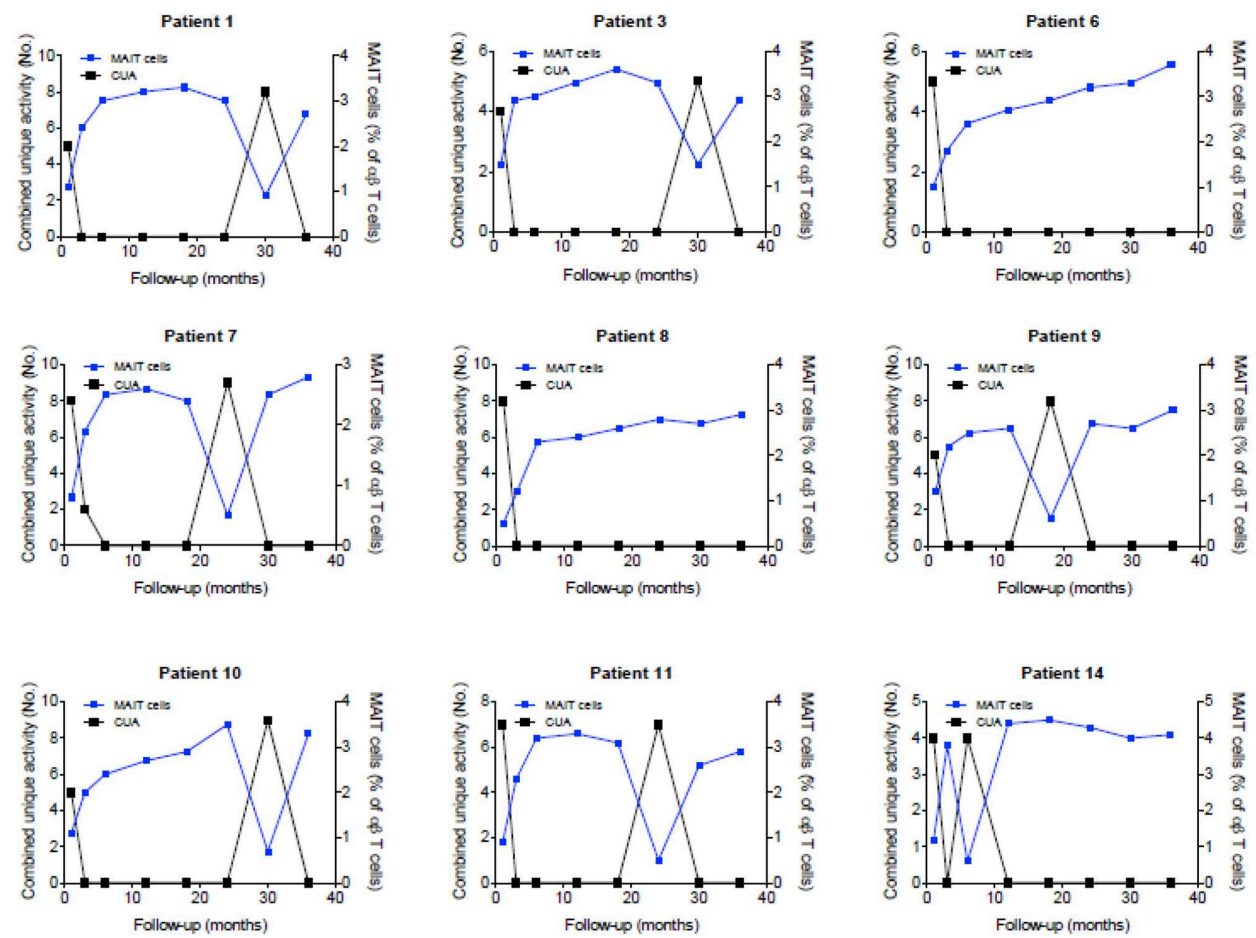

FIGURE 4 | MAIT cell percentages were related to disease activity. (A) After a clinical relapse and during follow-up (3-36 months), MAIT cell numbers were significantly increased, in parallel with clinical recovery, with high values persisting while disease remained stable. Each symbol represents values from single individuals $(n=15)$ and the middle line represents the mean. (B) During follow-up, seven patients examined at $6,18,24$, and 30 months showed further 
FIGURE 4 | decline in MAIT cell numbers (patients numbers 1, 3, 7, 9, 10, 11, and 14), while simultaneously showing disease-related MRI activity (A, closed circles), evidenced as new or enlarging T2 lesion and/or Gd-enhancing lesions on brain MRI screening; i.e., inverse correlation was observed between MAIT cell percentage and presence of new or enlarging T2 lesions, Gd-enhancing lesions, or CUA score. In contrast, in MS patients in whom MAIT cell numbers remained elevated during follow-up, there was no evidence of new MRI activity (patients 6 and 8). All patients studied received IFN- $\beta$ 1a treatment from the beginning of the study was seen. CUA: combined unique activity was calculated by adding new or enlarging T2 lesions to gadolinium-enhancing lesion. Correlations were performed using Spearman's correlation analysis.
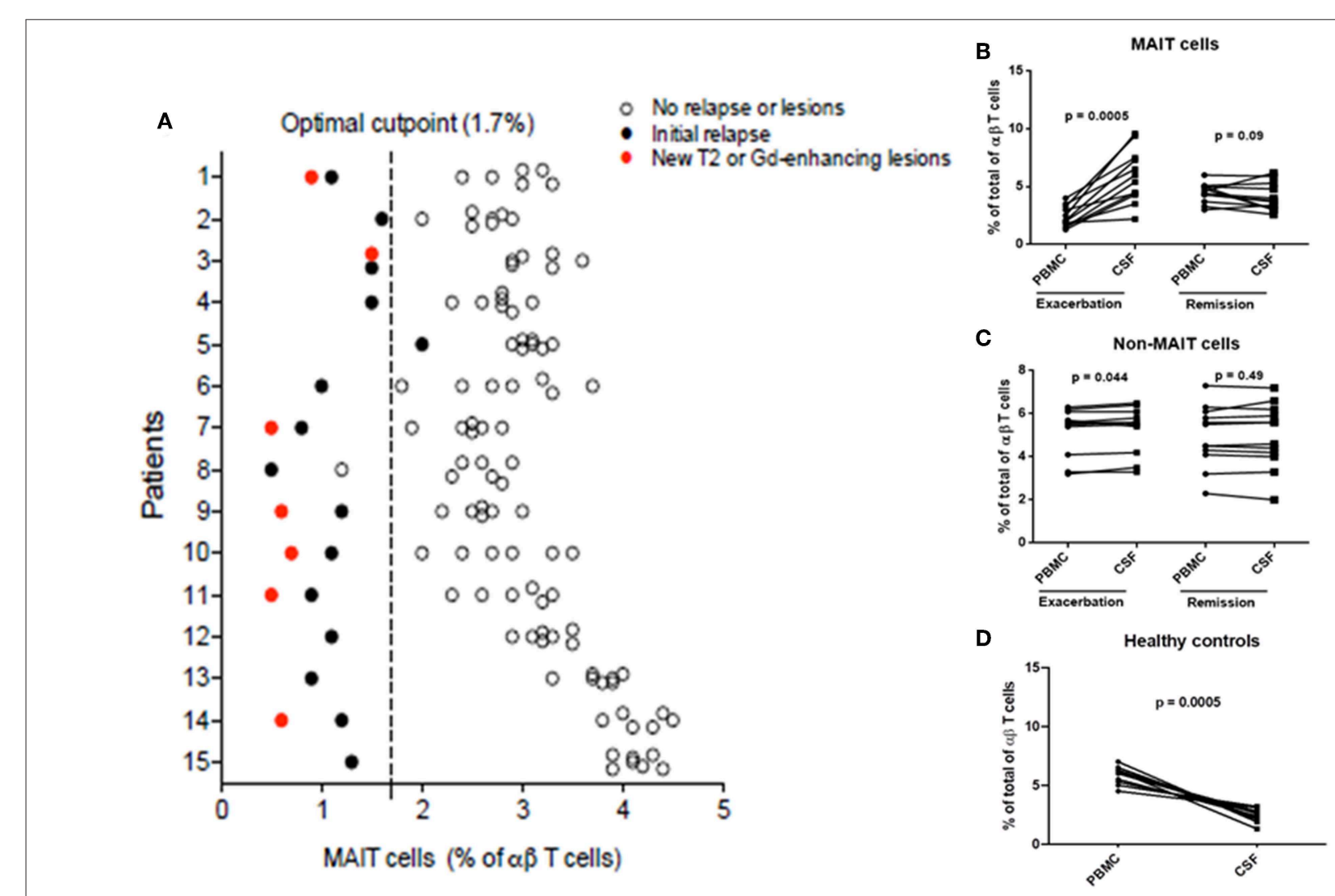

FIGURE 5 | Percentage of MAIT cells pred1icted disease activity. (A) Application of a predictive model defining clinical or MRI disease activity as a function of MAIT cell percentage demonstrated a cutoff point of 1.7. (B) Decreased circulating MAIT cells in MS were associated with recruitment to the CNS. MAIT cell numbers were significantly higher in CSF than in peripheral blood (paired samples) from 12 RRMS patients during exacerbations $(p=0.0005)$. However, no differences in MAIT cell percentage were found between peripheral blood and CSF in 12 RRMS patients in remission $(p=0.09)$. (C) Percentages of non-MAIT cells were also studied in the same group of patients and found to be slightly increased in CSF compared to peripheral blood during MS exacerbations $(p=0.044)$, but not during remissions $(p=$ 0.49). (D) Conversely, significantly lower numbers of MAIT cells were found in CSF compared to peripheral blood from healthy controls $(p=0.0005)$. For figures (B-D), statistical analysis was performed using the Wilcoxon matched-pairs signed rank test. PBMC: peripheral blood mononuclear cells, CSF: cerebrospinal fluid.

We next analyzed whether oligoclonality observed in circulating and CSF MAIT cells from RRMS patients remained stable over time. To address this issue, MAIT cell clones were isolated from seven MS patients at 12-months intervals for 3 years, and CDR3 $\beta$ clonotypes were investigated. In every individual, most CDR3 $\beta$ clonotypes identified at study entry were still detectable 36 months later. Some additional clonotypes were detected but not constantly. Results suggest that MAIT cell repertoires in peripheral blood remained stable over the study period (S4 Table).

\section{DISCUSSION}

Current advances in immunology have identified a novel population of innate immune $\mathrm{T}$ cells, called MAIT cells (8).
Their implication in MS pathogenesis remains elusive, but pro-inflammatory and homing patterns of these cells suggest involvement in lesion-generating processes $(10,22)$. Decoding whether MAIT cells participate in disease is important to understand MS pathophysiology and eventually develop new therapeutic strategies.

Studies have identified, in mice, clonally expanded $\mathrm{T}$ cells expressing the invariant TRAV1-TRJ33 TCR chain, which is highly homologous to the TRAV1-2-TRJ33 TCR chain described in human MAIT cells (7). As MAIT cells in mice are scarce (38), a transgenic ( $\mathrm{Tg})$ animal for the MAIT cellspecific TCR $\alpha$ chain (TRAV1-TRJ33) was generated (28). T cells expressing the TRAV1-TRJ33 TCR inhibited induction and progression of EAE, associated with decreased production of Th1 cytokines, and increase of IL-10, occurring at least in 

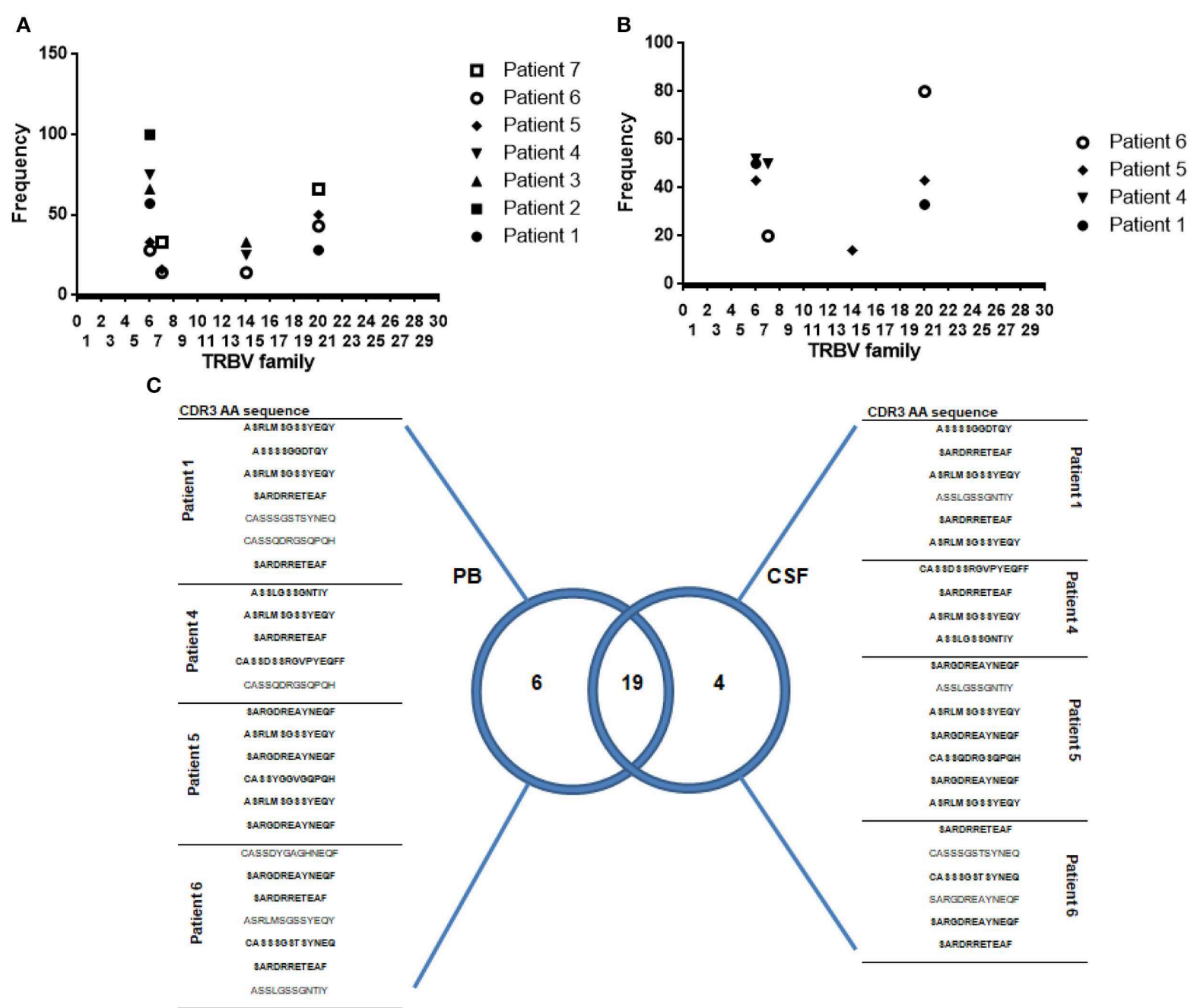

FIGURE 6 | Oligoclonality is a shared feature of both CSF and circulating MAIT cells. (A,B) Frequency of V $\beta$ usage by MAIT cell clones (TRAV1-2 ${ }^{+}$CD161 high) isolated from peripheral blood and CSF. Each symbol represents data from an individual MS patient. (C) Venn diagram showing distribution of unique and overlapping TRBV families, selected according to DNA encoding CDR3 $\beta$ amino acid sequences, from peripheral blood and CSF paired samples isolated from four MS patients during relapse. A total of twenty-five MAIT cell clones isolated from peripheral blood and 23 MAIT cell clones isolated from CSF were analyzed. CDR3 $\beta$ amino acid sequences marked in bold represent those common to both PB (peripheral blood) and CSF. TRBV: According to International ImMunoGeneTics information system ${ }^{\circledR}$ standardized nomenclature.

part through interaction between B cells and TRAV1-TRJ33 T cells (28).

Reduced numbers of circulating MAIT cells could reflect increased cell death due to sustained activation, or alternatively, greater recruitment toward the inflamed tissues. In the present study, we demonstrated that MAIT cells from MS patients, particularly during exacerbations, expressed high levels of the chemokine receptors CCR5, CCR6, and CXCR6, as well as of the integrin VLA-4. The specific phenotype of MAIT cells from MS patients suggests that they are more prone to migrate to the inflamed CNS. MAIT cell numbers from paired samples of peripheral blood and CSF from RRMS patients were significantly higher in CSF, supporting the theory of MAIT cell migration through the blood-brain barrier (BBB). These findings are consistent with observations in other autoimmune diseases such as type 1 diabetes (T1D), auto-inflammatory intestinal diseases, systemic lupus erythematosus, and rheumatoid arthritis, in which there is depletion of MAIT cells in the periphery and increase in affected tissues (39-42). In agreement with our findings, decreased numbers of circulating MAIT cells and infiltration into the CNS has already been described in RRMS patients by other authors $(23,26)$. However, in contrast to these results, there have been variable reports on frequencies of MAIT cells in the circulation of RRMS patients compared to controls, with some reporting increased frequencies, no changes in frequencies, or a selective increase in IL-17 ${ }^{+}$CCR6 $^{+}$MAIT cells $(25,27,43-$ 45). Interestingly, increased IL-17 production by MAIT cells is very likely not just a sign of augmented activation, but rather represents a more pronounced differentiation toward a type-17 phenotype in MS patients, linked to IL-7 signaling (45). Different 


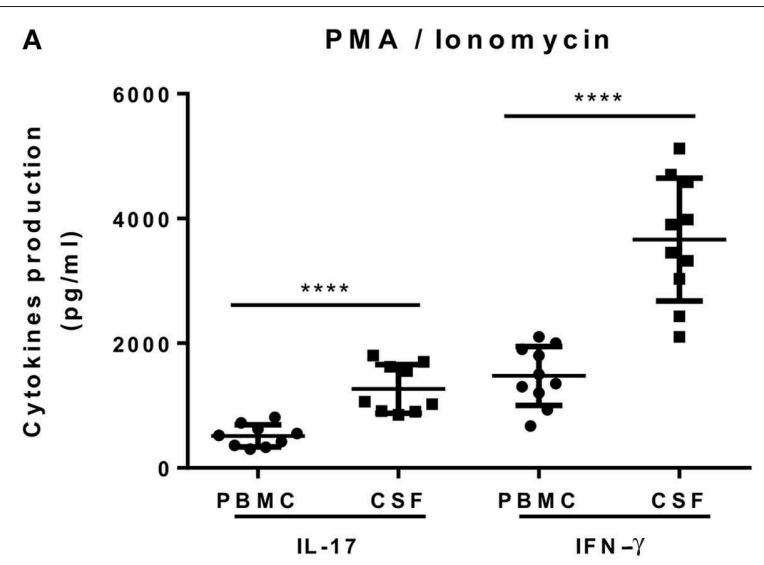

C

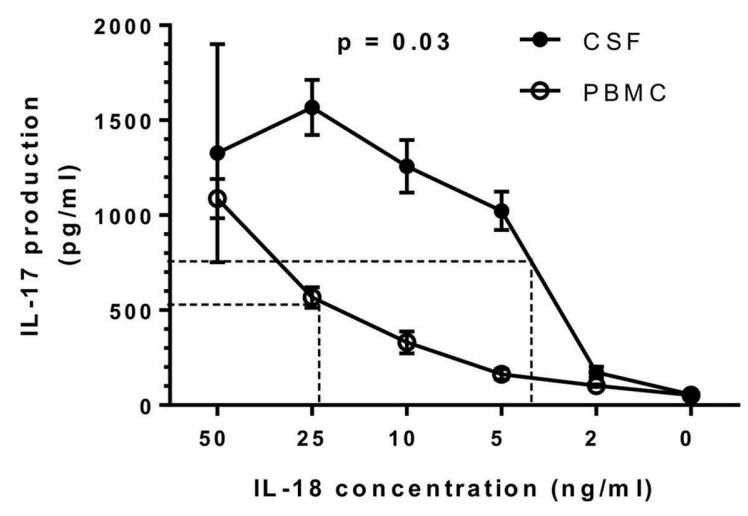

B

\section{E.Coli-HeLa}

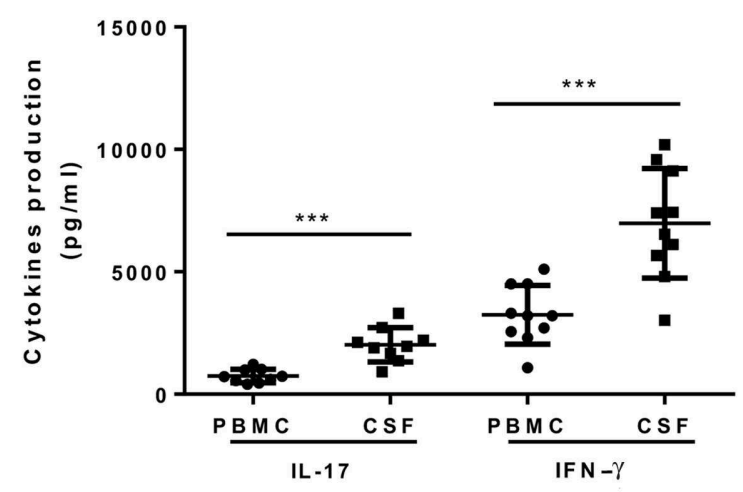

D

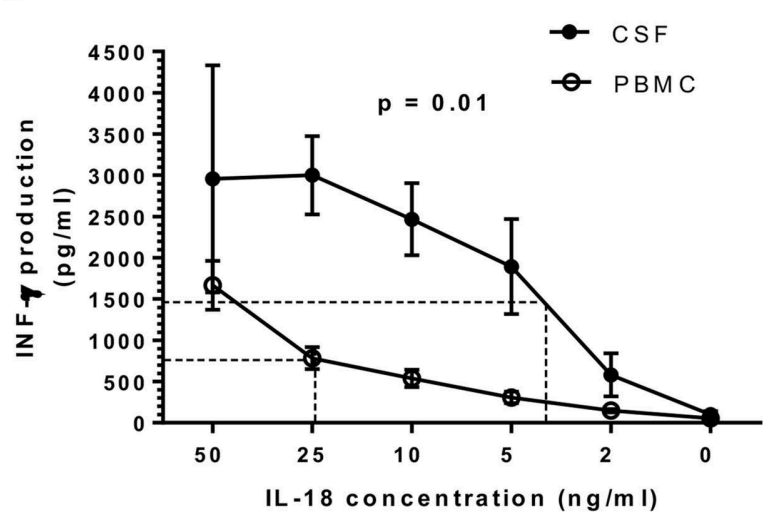

FIGURE 7 | IL-17 and IFN- $\gamma$ secretion by CSF MAIT cell clones is significantly higher compared to that of peripheral blood MAIT cell clones. (A) CSF ( $n=19)$ and peripheral blood ( $n=19$ ) MAIT cell clones were stimulated with PMA/ionomycin and IL-17 $(n=9)$, after which IFN- $\gamma(n=10)$ was assessed in the supernatants using ELISA. (B) Both groups of MAIT cell clones were stimulated in a second protocol using $E$. coli-infected HeLa cells and cytokines assessed in a similar manner. (C,D) $\mathrm{IL}-18$ was titrated in CSF and peripheral blood MAIT cell clones and secretion of IL-17 and IFN- $\gamma$ was assessed using ELISA. Both graphics show that CSF MAIT cell clones are more sensitive to IL-18 stimulation than MAIT cell clones isolated from peripheral blood. In panel (D), IL-18 was titrated in the presence of IL-12 (10 ng/ml). Data represent mean \pm SEM of three different MAIT cell clones for each condition. Dotted lines indicate IL-18 concentrations necessary to reach $50 \%$ of maximum secretion for each cytokine. Statistical analysis was performed using Mann-Whitney $U$ test $(\mathbf{A}, \mathbf{B})$ and Wilcoxon matched-pairs signed rank test $(\mathrm{C}, \mathrm{D}) .{ }^{\star \star \star} p<0.001$, ${ }^{\star \star \star \star} p<0.0001$.

factors could explain these discrepancies. First, differences in corticosteroid treatment in particular may hamper valid cohort comparisons. In this study, no patients had received steroid or immunosuppressant treatments, for at least 6 months prior to entry. Second, MAIT cell frequency has been shown to decrease with age (46). In our study, no significant differences in age were found between RRMS or PPMS patients and their respective control groups. Therefore, age would not seem to be a determining factor, or explain differences found in peripheral blood MAIT cell numbers between these cohorts. Nevertheless, children with MS showed abnormally increased frequencies and exaggerated pro-inflammatory responses of MAIT cells, which was associated with a relative resistance to suppression by normal Treg cells (43). Third, MAIT cells provoke specific immune responses to distinct microorganisms, in varying magnitude, despite a limited known diversity in riboflavin metabolite antigens (47). The fact that different cohorts come from different countries, exposed to different environmental factors, and especially to varying gut microbiota, could be critical. Finally, methodological differences between studies, particularly differences in gating strategies, could justify the discrepancies observed (27).

Controversy also exists over how MAIT cell recruitment into the CNS through CSF occurs. One early study had shown that although MAIT cells are capable of infiltrating the CNS and expressing VLA-4 (a molecule essential for crossing the $\mathrm{BBB})$, MAIT cells were extremely rare in CSF and present in extremely small numbers compared to those in blood from the same patients (23). The authors suggested that infiltration into the CNS was a consequence of migration through parenchymal vessels and not through the $\mathrm{BBB}$, although they could not entirely rule out $\mathrm{CD}^{+}$MAIT cell entry through the subarachnoid space. In another study (27), transmigratory capacity of MAIT cells was evaluated through an in vitro assay using the human 
brain endothelial cell line hCMEC/CD3, which mimics BBB features. (48) Clearly, methodological differences between both studies do not allow valid comparisons. The interaction between cell adhesion molecules expressed by endothelial cells of the BBB and their cognate ligands, present on activated leukocytes, plays a central role in transmigration of immune cells into the CNS. In addition, chemokines present on the luminal surface of the endothelium can trigger integrin-dependent adhesion of leukocytes, previously crawling toward interendothelial junctions (49). Similarities between phenotypes of adhesion molecules and chemokines present in conventional $\mathrm{T}$ cells and MAIT cells suggest that MAIT cells can migrate into the CNS through a similar route. However, MAIT cells have high expression of adhesion molecules and chemokine receptors even in the remission phase. Nevertheless, the recruitment of MAIT cells to the CNS was different in remission vs. exacerbations. It is possible that, in our study, not all lymphocyte transmigration molecules have been studied, including activated leukocyte cell adhesion molecule (ALCAM), melanoma adhesion molecule (MCAM), and laminin-411. Subpopulations of activated $\mathrm{CD} 4^{+} \mathrm{T}$ cells producing increased levels of IL-17 and GM-CSF preferentially migrate across the BBB compared to cells devoid of these molecules (50-52), suggesting that they facilitate cell entry of pro-inflammatory cytokines producing cells into the CNS during active pathology. Alternatively, MAIT cells during exacerbations can migrate into the CNS by two additional routes: the bloodCSF barrier in the choroid plexus and through meningeal arteries (53). Therefore, more specific studies will be needed to address how MAIT migration patterns are fine-tuned across the BBB.

Decrease in circulating MAIT cell frequency in MS was associated with increased pro-inflammatory cytokine production, particularly IFN- $\gamma$, GM-CSF, TNF- $\alpha$, and IL-17, compared to healthy donors. All these cytokines are considered major players in many autoimmune diseases, including MS. Therefore, contrary to what was observed in mice, infiltrating MAIT cells in MS subjects are probably pro-inflammatory cells causing deleterious effects. Functional differences between TRAV1-Tg mice and human MAIT cells could be explained by differences in MAIT cell development. While Tg mouse MAIT cells displayed a naïve phenotype, MAIT cells in human adults show a memory/effector-like phenotype (54).

Most previous studies on the role of MAIT cells in MS had cross-sectional design, or very short patient follow-up. In the present study, a subgroup of RRMS patients were followed longitudinally for more than 3 years and circulating MAIT cell numbers were linked to clinical and radiological disease activity. Our results show that MS exacerbations were associated with significant reduction in circulating MAIT cells. Interestingly, when blood samples were analyzed 3 months later, circulating MAIT cell numbers were significantly increased in line with clinical recovery, a finding also reflected by dampened MRI disease activity. Decreased circulating MAIT cell numbers observed in some patients during the course disease were also associated with increased MRI activity. Even though the number of MS subjects followed in this study was limited, results nevertheless suggest that MAIT cell levels reflect disease activity, not only playing an active role in
MS pathogenesis, but also possibly representing a potential biomarker of disease.

As previously mentioned, MAIT cell traffic between CSF and peripheral blood remains poorly characterized, although it has recently been shown that B-cell clones are shared between CNS, CSF, and peripheral blood in MS patients, with clonal diversification occurring in all three compartments (55). Likewise, CD8 clones identified in brain biopsies have also been found in CSF and blood (56). Our results indicate similar CDR3 $\beta$ chain oligoclonality in MAIT cells from both peripheral blood and CSF from RRMS patients. However, when the oligoclonality observed in peripheral blood and CSF from healthy subjects was analyzed separately, contrary to what was found in RRMS patients, CDR3 $\beta$ clonotypes observed in paired samples of peripheral blood and CSF differed significantly in individual subjects (S3 Table). Furthermore, a greater number of MAIT cell clones capable of producing pro-inflammatory cytokines (IL-17, IFN- $\gamma$, and GM-CSF) were identified in both peripheral blood and CSF from patients with RRMS compared to control subjects. In addition, frequency of blood MAIT cells inversely correlated with clinical and MRI disease activity, suggesting that MAIT cells may be involved in MS pathogenesis, and levels found in peripheral blood could reflect inflammatory events within the CNS. Of note, CSF MAIT cell clones produced significantly higher amounts of IL-17 and IFN- $\gamma$ than peripheral blood MAIT cell clones isolated from the same patients and were more readily stimulated by IL-18, further reinforcing the notion that MAIT cells play a harmful role in MS.

Our results also indicated that peripheral blood MAIT cell repertoires remained stable over time, in agreement with observations from longitudinal studies in which MAIT cells massively expanded in CNS of MS patients at onset, persisted for several years in blood or CSF, and were still being detectable in blood after 18 years (22).

The absence of MAIT cells in the intestinal lamina propria of germ-free mice (11) and their localization under physiological conditions at various mucosal sites, including the intestine, underscores the role of the commensal intestinal microbiota in the expansion of MAIT cells and survival in the periphery. Based on observations from murine models, MAIT cells appear to leave the thymus as näive cells, and then encounter commensal bacterial antigens that drive their maturation, in the periphery (57). This is not however the case in human fetal mucosal tissue of the small intestine, lung, and liver, which have a population of MAIT cells that exhibit a phenotype consistent with mature MAIT cells, with high proliferation capacity. These observations indicate that, in humans, MAIT cells acquire an antimicrobial responsiveness in mucosal tissues before exposure to environmental microbes and the commensal microflora (58). Changes in gut microbiota composition occurring during MS (59) may impact gut permeability, and presence of bacterial ligands may activate MAIT cells, although importance of this interaction needs to be further explored. Alternatively, the presence of endogenous ligands induced during autoinflammatory processes could regulate MAIT cell activation.

Overall, MAIT cells are a relatively new class of unconventional $\mathrm{T}$ cell. Their discovery has opened new avenues 
for the study of autoimmune disease pathogenesis, including that of MS. Correlation with MS activity and alterations prior to T1D onset (60) suggest that MAIT cells could represent a new biomarker for certain autoimmune diseases. Further investigations are warranted on the link between MS, MAIT cell function, and gut microbiota. Finally, discovery of MAIT cell inhibitors (17) may pave the way for the development of new and more promising therapeutic strategies.

\section{DATA AVAILABILITY STATEMENT}

The raw data supporting the conclusions of this manuscript will be made available by the authors, without undue reservation, to any qualified researcher.

\section{ETHICS STATEMENT}

This study was reviewed and approved by the local Institutional Ethics Committee (FLENI), and written informed consent was obtained from all participants.

\section{AUTHOR CONTRIBUTIONS}

JC: conceptualization, funding acquisition, methodology, project administration, resources, supervision, and validation. JC, EC, and MF: data curation, investigation, visualization, and writing-review and editing. JC and EC: writing-original draft.

\section{REFERENCES}

1. International Multiple Sclerosis Genetics Consortium, Wellcome Trust Case Control Consortium 2, Sawcer S, Hellenthal G, Pirinen M, Spencer CC, et al. Genetic risk and a primary role for cell-mediated immune mechanisms in multiple sclerosis. Nature. (2011) 476:214-9. doi: 10.1038/nature10251

2. Henderson AP, Barnett MH, Parratt JD, Prineas JW. Multiple sclerosis: distribution of inflammatory cells in newly forming lesions. Ann Neurol. (2009) 66:739-53. doi: 10.1002/ana.21800

3. Hemmer B, Kerschensteiner M, Korn T. Role of the innate and adaptive immune responses in the course of multiple sclerosis. Lancet Neurol. (2015) 14:406-19. doi: 10.1016/S1474-4422(14)70305-9

4. Sospedra M, Martin R. Immunology of multiple sclerosis. Semin Neurol. (2016) 36:115-27. doi: 10.1055/s-0036-1579739

5. Bianchini E, De Biasi S, Simone AM, Ferraro D, Sola P, Cossarizza $\mathrm{A}$, et al. Invariant natural killer $\mathrm{T}$ cells and mucosal-associated invariant $\mathrm{T}$ cells in multiple sclerosis. Immunol Lett. (2017) 183:1-7. doi: 10.1016/j.imlet.2017.01.009

6. Mohammadi H, Sharafkandi N, Hemmatzadeh M, Azizi G, Karimi M, JadidiNiaragh F, et al. The role of innate lymphoid cells in health and disease. J Cell Physiol. (2018) 233:4512-29. doi: 10.1002/jcp.26250

7. Tilloy F, Treiner E, Park SH, Garcia C, Lemonnier F, de la Salle H, et al. An invariant $\mathrm{T}$ cell receptor alpha chain defines a novel TAP-independent major histocompatibility complex class Ib-restricted alpha/beta T cell subpopulation in mammals. J Exp Med. (1999) 189:1907-21. doi: 10.1084/jem.189. 12.1907

8. Porcelli S, Yockey CE, Brenner MB, Balk SP. Analysis of T cell antigen receptor (TCR) expression by human peripheral blood CD4-8- alpha/beta $\mathrm{T}$ cells demonstrates preferential use of several $\mathrm{V}$ beta genes and an invariant TCR alpha chain. J Exp Med. (1993) 178:1-16. doi: 10.1084/jem. 178.1.1

\section{SUPPLEMENTARY MATERIAL}

The Supplementary Material for this article can be found online at: https://www.frontiersin.org/articles/10.3389/fimmu. 2019.02690/full\#supplementary-material

Supplementary Figure S1 | (A) Frequency and (B) absolute numbers of total, $\mathrm{CD}^{+}$, and DN MAIT cells in peripheral blood, from healthy controls, RRMS patients in remission, RRMS patients during exacerbations, and PPMS patients. Data are presented as mean values \pm SEM from seven different experiments. Statistical analysis was performed using the Wilcoxon matched-pairs signed rank test. ${ }^{* *} p<0.01,{ }^{* * *} p<0.001,{ }^{* * * *} p<0.0001$.

Supplementary Figure S2 | (A-C) Expression of CCR5, CCR6, and CXCR6 in both MAIT and non-MAIT cells. (D-G) Expression of integrins and adhesion molecules VLA-4, LFA-1, PSGL-1, and LFA-3 in both MAIT and non-MAIT cells. In all cases data are presented as mean values \pm SEM from 24 RRMS patients (MS in remission = 12 and MS during exacerbations = 12), 12 PPMS patients and 12 healthy controls. Statistical analysis was performed using the Wilcoxon matched-pairs signed rank test (A-D). ${ }^{*} p<0.05,{ }^{* *} p<0.01,{ }^{* * *} p<0.001$.

Supplementary Figure S3 | Correlations between MAIT cell numbers and MRI lesions estimated using Spearman's correlation. (A) New Gd+ lesions. (B) New or enlarging T2 lesions. (C) CUA score.

S1 Table | Phenotype and TCR $\beta$ amino acid sequences of MAIT cell clones isolated from blood of RRMS patients at the beginning of the study.

S2 Table | Phenotype and TCR $\beta$ amino acid sequences of MAIT cell clones isolated from CSF of RRMS patients.

S3 Table | Phenotype and TCR $\beta$ amino acid sequences of MAIT cell clones isolated from peripheral blood and CSF from healthy controls.

S4 Table | Longitudinal analysis of the TCR $\beta$ chain sequence frequency in clones of MAIT cells isolated from patients with RRMS over time.

9. Lepore M, Kalinichenko A, Colone A, Paleja B, Singhal A, Tschumi A, et al. Parallel T-cell cloning and deep sequencing of human MAIT cells reveal stable oligoclonal TCR $\beta$ repertoire. Nat Commun. (2014) 5:3866. doi: $10.1038 /$ ncomms5493

10. Treiner E, Liblau RS. Mucosal-associated invariant $T$ cells in multiple sclerosis: the jury is still out. Front Immunol. (2015) 6:503. doi: 10.3389/fimmu.2015.00503

11. Treiner E, Duban L, Bahram S, Radosavljevic M, Wanner V, Tilloy F, et al. Selection of evolutionarily conserved mucosal-associated invariant $\mathrm{T}$ cells by MR1. Nature. (2003) 422:164-9. doi: 10.1038/nature01433

12. Kjer-Nielsen L, Patel O, Corbett AJ, Le Nours J, Meehan B, Liu L, et al. MR1 presents microbial vitamin B metabolites to MAIT cells. Nature. (2012) 491:717-23. doi: 10.1038/nature11605

13. Eckle SB, Corbett AJ, Keller AN, Chen Z, Godfrey DI, Liu L, et al. Recognition of vitamin B precursors and byproducts by mucosal associated invariant T cells. J Biol Chem. (2015) 290:30204-11. doi: 10.1074/jbc.R115.6 85990

14. Lepore M, Kalinichenko A, Calogero S, Kumar P, Paleja B, Schmaler M, et al. Functionally diverse human $\mathrm{T}$ cells recognize non-microbial antigens presented by MR1. Elife. (2017) 18:6. doi: 10.7554/eLife.24476

15. Kurioka A, Ussher JE, Cosgrove C, Clough C, Fergusson JR, Smith $\mathrm{K}$, et al. MAIT cells are licensed through granzyme exchange to kill bacterially sensitized targets. Mucosal Immunol. (2015) 8:429-40. doi: $10.1038 / \mathrm{mi} .2014 .81$

16. Le Bourhis L, Martin E, Pégillet I, Guihot A, Froux N, Coré M, et al. Antimicrobial activity of mucosal-associated invariant T cells. Nat Immunol. (2010) 11:701-8. doi: 10.1038/ni.1890

17. Le Bourhis L, Dusseaux M, Bohineust A, Bessoles S, Martin E, Premel $\mathrm{V}$, et al. MAIT cells detect and efficiently lyse bacterially-infected epithelial cells. PLoS Pathog. (2013) 9:e1003681. doi: 10.1371/journal.ppat.1 003681 
18. Leeansyah E, Svärd J, Dias J, Buggert M, Nyström J, Quigley MF, et al. Arming of MAIT cell cytolytic antimicrobial activity is induced by IL7 and defective in HIV-1 infection. PLoS Pathog. (2015) 11:e1005072. doi: 10.1371/journal.ppat.1005072

19. Xiao X, Cai J. Mucosal-associated invariant $\mathrm{T}$ cells: new insights into antigen recognition and activation. Front Immunol. (2017) 8:1540. doi: 10.3389/fimmu.2017.01540

20. Dusseaux M, Martin E, Serriari N, Péguillet I, Premel V, Louis D, et al. Human MAIT cells are xenobiotic-resistant, tissue-targeted, CD161hi IL-17-secreting T cells. Blood. (2011) 117:1250-9. doi: 10.1182/blood-2010-08-303339

21. Billerbeck E, Kang YH, Walker L, Lockstone H, Grafmueller S, Fleming V, et al. Analysis of CD161 expression on human CD8+ T cells defines a distinct functional subset with tissue-homing properties. Proc Natl Acad Sci USA. (2010) 107:3006-11. doi: 10.1073/pnas.0914839107

22. Held K, Bhonsle-Deeng L, Siewert K, Sato W, Beltrán E, Schmidt S, et al. $\alpha \beta$ T-cell receptors from multiple sclerosis brain lesions show MAIT cell-related features. Neurol Neuroimmunol Neuroinflamm. (2015) 2:e107. doi: 10.1212/NXI.0000000000000107

23. Willing A, Leach OA, Ufer F, Attfield KE, Steinbach K, Kursawe N, et al. CD8 MAIT cells infiltrate into the CNS and alterations in their blood frequencies correlate with IL-18 serum levels in multiple sclerosis. Eur J Immunol. (2014) 44:3119-28. doi: 10.1002/eji.201344160

24. Illés Z, Shimamura M, Newcombe J, Oka N, Yamamura T. Accumulation of Valpha7.2-Jalpha33 invariant $\mathrm{T}$ cells in human autoimmune inflammatory lesions in the nervous system. Int Immunol. (2004) 16:223-30 doi: 10.1093/intimm/dxh018

25. Annibali V, Ristori G, Angelini DF, Serafini B, Mechelli R, Cannoni S, et al. CD161(high)CD8+T cells bear pathogenetic potential in multiple sclerosis. Brain. (2011) 134(Pt 2):542-54. doi: 10.1093/brain/awq354

26. Miyazaki Y, Miyake S, Chiba A, Lantz O, Yamamura T. Mucosal-associated invariant $\mathrm{T}$ cells regulate $\mathrm{Th} 1$ response in multiple sclerosis. Int Immunol. (2011) 23:529-35. doi: 10.1093/intimm/dxr047

27. Salou M, Nicol B, Garcia A, Baron D, Michel L, Elong-Ngono A, et al. Neuropathologic, phenotypic and functional analyses of mucosal associated invariant T cells in multiple sclerosis. Clin Immunol. (2016) 166-7:1-11. doi: 10.1016/j.clim.2016.03.014

28. Croxford JL, Miyake S, Huang YY, Shimamura M, Yamamura T. Invariant $\mathrm{V}$ (alpha)19i T cells regulate autoimmune inflammation. Nat Immunol. (2006) 7:987-94. doi: 10.1038/ni1370

29. Løken-Amsrud KI, Holmøy T, Bakke SJ, Beiske AG, Bjerve KS, Bjørnarå $\mathrm{BT}$, et al. Vitamin $\mathrm{D}$ and disease activity in multiple sclerosis before and during interferon- $\beta$ treatment. Neurology. (2012) 79:267-73. doi: 10.1212/WNL.0b013e31825fdf01

30. Kraan J, Gratama JW, Haioun C, Orfao A, Plonquet A, Porwit A, et al. et al. Flow cytometric immunophenotyping of cerebrospinal fluid. Curr Protoc Cytom. (2008) 45:6.25.1-16. doi: 10.1002/0471142956.cy0 $625 s 45$

31. Meermeier E, Laugel B, Sewell A.K, Corbett AJ, Rossjohn J, McCluskey J, et al. Human TRAV1-2-negative MR1-restricted T cells detect S. pyogenes and alternatives to MAIT riboflavin-based antigens. Nat Commun. (2016) 7:12506. doi: $10.1038 /$ ncomms 12506

32. Correale J, Rush C, Amengual A, Goicochea MT. Mitoxantrone as rescue therapy in worsening relapsing-remitting MS patients receiving IFNbeta. J Neuroimmunol. (2005) 162:173-83. doi: 10.1016/j.jneuroim.2005. 02.003

33. Dias J, Sobkowiak MJ, Sandberg JK, Leeansyah E. Human MAITcell responses to Escherichia coli: activation, cytokine production, proliferation, and cytotoxicity. J Leukoc Biol. (2016) 100:233-40. doi: 10.1189/jlb.4TA0815-391RR

34. Robins HS, Campregher PV, Srivastava SK, Wacher A, Turtle CJ, Kahsai O, et al. Comprehensive assessment of T-cell receptor betachain diversity in alphabeta $\mathrm{T}$ cells. Blood. (2009) 114:4099-107. doi: 10.1182/blood-2009-04-217604

35. Dias J, Boulouis C, Gorin JB, van den Biggelaar RHGA, Lal KG, Gibbs A, et al. The CD4-CD8- MAIT cell subpopulation is a functionally distinct subset developmentally related to the main CD8+ MAIT cell pool. Proc Natl Acad Sci USA. (2018) 115:E11513-22. doi: 10.1073/pnas.18122 73115
36. Ussher JE, Bilton M, Attwod E, Shadwell J, Richardson R, de Lara C, et al. $\mathrm{CD} 161++\mathrm{CD} 8+\mathrm{T}$ cells, including the MAIT cell subset, are specifically activated by IL-12+IL-18 in a TCR-independent manner. Eur J Immunol. (2014) 44:195-203. doi: 10.1002/eji.201343509

37. Balashov KE, Rottman JB, Weiner HL, Hancock WW. CCR5(+) and CXCR3(+) T cells are increased in multiple sclerosis and their ligands MIP1alpha and IP-10 are expressed in demyelinating brain lesions. Proc Natl Acad Sci USA. (1999) 96:6873-8. doi: 10.1073/pnas.96.12.6873

38. Rahimpour A, Koay HF, Enders A, Clanchy R, Eckle SB, Meehan B, et al. Identification of phenotypically and functionally heterogeneous mouse mucosal-associated invariant T cells using MR1 tetramers. J Exp Med. (2015) 212:1095-108. doi: 10.1084/jem.20142110

39. Serriari NE, Eoche M, Lamotte L, Lion J, Fumery M, Marcelo P, et al. Innate mucosal-associated invariant T (MAIT) cells are activated in inflammatory bowel diseases. Clin Exp Immunol. (2014) 176:266-74. doi: 10.1111/cei.12277

40. Haga K, Chiba A, Shibuya T, Osada T, Ishikawa D, Kodani T, et al. MAIT cells are activated and accumulated in the inflamed mucosa of ulcerative colitis. $J$ Gastroenterol Hepatol. (2016) 31:965-72. doi: 10.1111/jgh.13242

41. Magalhaes I, Pingris K, Poitou C, Bessoles S, Venteclef N, Kiaf B, et al. Mucosal-associated invariant $\mathrm{T}$ cell alterations in obese and type 2 diabetic patients. J Clin Invest. (2015) 125:1752-62. doi: 10.1172/JCI78941

42. Cho YN, Kee SJ, Kim TJ, Jin HM, Kim MJ, Jung HJ, et al. Mucosal-associated invariant $\mathrm{T}$ cell deficiency in systemic lupus erythematosus. J Immunol. (2014) 193:3891-901. doi: 10.4049/jimmunol.1302701

43. Mexhitaj I, Nyirenda MH, Li R, O'Mahony J, Rezk A, Rozenberg A, et al. Abnormal effector and regulatory $\mathrm{T}$ cell subsets in paediatric-onset multiple sclerosis. Brain. (2019) 142:617-32. doi: 10.1093/brain/awz017

44. Negrotto L, Canto E, Río J, Tintore M, Montalban X, Comabella M. Peripheral blood non-MAIT CD8+ 161 hi cells are decreased in relapsing-remitting multiple sclerosis patients treated with interferon beta. J Neuroimmunol. (2015) 288:98-101. doi: 10.1016/j.jneuroim.2015.09.007

45. Willing A, Jäger J, Reinhardt S, Kursawe N, Friese MA. Porduction of IL-17 by MAIT cells is increased in multiple sclerosis and is associated with IL-7 receptor expression. J immunol. (2018) 200:974-82. doi: $10.4049 /$ jimmunol.1701213

46. Novak J, Dobrovolny J, Novakova L, Kozak T. The decrease in number and change in phenotype of mucosal-associated invariant $\mathrm{T}$ cells in the elderly and differences in men and women of reproductive age. Scand J Immunol. (2014) 80:271-5. doi: $10.1111 /$ sji. 12193

47. Dias J, Leeansyah E, Sandberg JK. Multiple layers of heterogeneity and subset diversity in human MAIT cell responses to distinct microorganisms and to innate cytokines. Proc Natl Acad Sci USA. (2017) 114:E5434-43. doi: 10.1073/pnas.1705759114

48. Weksler BB, Subileau EA, Perriere N, Charneau P, Holloway K, Laveque $\mathrm{M}$, et al. Blood-brain barrier-specific properties of a human adult brain endotelial cell line. FASEB J. (2005) 19:1872-4. doi: 10.1096/fj.04-3 458 fje

49. Takeshita Y, Ransohoff RM. Inflammatory cell trafficking across the bloodbrain barrier: chemokine regulation and in vitro models. Immunol Rev. (2012) 248:228-39. doi: 10.1111/j.1600-065X.2012.01127.x

50. Larochelle C, Cayrol R, Kebir H, Alvarez JI, Lécuyer MA, Ifergan I, et al. Melanoma cell adhesion molecule identifies encephalitogenic T lymphocytes and promotes their recruitment to the central nervous system. Brain. (2012) 135:2906-24. doi: 10.1093/brain/aws212

51. Flanagan K, Fitzgerald K, Baker J, Regnstrom K, Gardai S, Bard F, et al. Laminin-411 is a vascular ligand for MCAM and facilitates TH17 cell entry into the CNS. PLoS ONE. (2012) 7:e40443. doi: 10.1371/journal.pone. 0040443

52. Schneider-Hohendorf $\mathrm{T}$, Rossaint $\mathrm{J}$, Mohan H, Böning D, Breuer J, Kuhlmann T, et al. VLA-4 blockade promotes differential routes into human CNS involving PSGL-1 rolling of T cells and MCAM-adhesion of TH17 cells. J Exp Med. (2014) 211:1833-46. doi: 10.1084/jem. 20140540

53. Engelhardt B, Vajkoczy P, Weller RO. The movers and shapers in immune privilege of the CNS. Nat Immunol. (2017) 18:123-31. doi: 10.1038/ni.3666

54. Martin E, Treiner E, Duban L, Guerri L, Laude H, Toly C, et al. Stepwise development of MAIT cells in mouse and human. PLoS Biol. (2009) 7:e54. doi: 10.1371/journal.pbio.1000054 
55. von Büdingen HC, Kuo TC, Sirota M, van Belle CJ, Apeltsin L, Glanville J, et al. B cell exchange across the blood-brain barrier in multiple sclerosis. $J$ Clin Invest. (2012) 122:4533-43. doi: 10.1172/JCI63842

56. Skulina C, Schmidt S, Dornmair K, Babbe H, Roers A, Rajewsky K, et al. Multiple sclerosis: brain-infiltrating CD8+ T cells persist as clonal expansions in the cerebrospinal fluid and blood. Proc Natl Acad Sci USA. (2004) 101:242833. doi: $10.1073 /$ pnas. 0308689100

57. Le Bourhis L, Mburu YK, Lantz O. MAIT cells, surveyors of a new class of antigen: development and functions. Curr Opin Immunol. (2013) 25:174-80. doi: 10.1016/j.coi.2013.01.005

58. Leeansyah E, Loh L, Nixon DF, Sandberg JK. Acquisition of innatelike microbial reactivity in mucosal tissues during human fetal MAIT-cell development. Nat Commun. (2014) 5:3143. doi: 10.1038/ ncomms 4143

59. Cekanaviciute E, Yoo BB, Runia TF, Debelius JW, Singh S, Nelson CA et al. Gut bacteria from multiple sclerosis patients modulate human $\mathrm{T}$ cells and exacerbate symptoms in mouse models. Proc Natl Acad Sci USA. (2017) 114:10713-8. doi: 10.1073/pnas.1711235114

60. Rouxel O, Da Silva J, Beaudoin L, Nel I, Tard C, Cagninacci L, et al. Cytotoxic and regulatory roles of mucosal-associated invariant T cells in type 1 diabetes. Nat Immunol. (2017) 18:1321-31. doi: 10.1038/ni.3854
Conflict of Interest: EC has received reimbursement for developing educational presentations, educational and research grants as well as professional travel/accommodation stipends from Biogen-Idec, Genzyme, Merck-Serono, Novartis, TEVA Bayer and Roche. MF has received professional travel accommodation stipends from Merck-Serono, TEVA, and Novartis and research funding from Biogen-Idec and Novartis. JC is a board member of Merck-Serono Argentina, Novartis Argentina, Genzyme LATAM, Genzyme global, Biogen-Idec LATAM, and Merck-Serono LATAM. He is on the Steering Committee for the Ofatumumab clinical trials (Novartis Global). JC has received reimbursement for developing educational presentations for Merck-Serono Argentina, MerckSerono LATAM, Biogen-Idec Argentina, Genzyme Argentina, Novartis Argentina, Novartis LATAM, Novartis Global, and TEVA Argentina as well as professional travel/accommodation stipends.

Copyright (c) 2019 Carnero Contentti, Farez and Correale. This is an open-access article distributed under the terms of the Creative Commons Attribution License (CC $B Y)$. The use, distribution or reproduction in other forums is permitted, provided the original author(s) and the copyright owner(s) are credited and that the original publication in this journal is cited, in accordance with accepted academic practice. No use, distribution or reproduction is permitted which does not comply with these terms. 